\begin{tabular}{|c|l|}
\hline Title & $\begin{array}{l}\text { Nonlinear propagation analysis of few-optical-cycle pul ses for subfemtosecond compression and carrier envelope phase } \\
\text { effect }\end{array}$ \\
\hline Author(s) & Mizuta, Yo; Nagasawa, Minoru; Ohtani, Morimasa; Y amashita, Mikio \\
\hline Citation & $\begin{array}{l}\text { Physical Review A, 72, 063802 } \\
\text { https://doi.org/10.1103/PhysRevA.72.063802 }\end{array}$ \\
\hline Issue Date & 2005-12-06 \\
\hline Doc URL & http://hdl.handle.net/2115/991 \\
\hline Rights & Copyright $\odot 2005$ The A merican Physical Society \\
\hline Type & article \\
\hline File Information & PRA 72.pdf \\
\hline
\end{tabular}

Instructions for use 


\title{
Nonlinear propagation analysis of few-optical-cycle pulses for subfemtosecond compression and carrier envelope phase effect
}

\author{
Yo Mizuta, ${ }^{*}$ Minoru Nagasawa, Morimasa Ohtani, and Mikio Yamashita \\ Department of Applied Physics, Faculty of Engineering, Hokkaido University, Kita-13, Nishi-8, Kita-ku, Sapporo 060-8628, Japan
}

(Received 7 December 2004; revised manuscript received 24 August 2005; published 6 December 2005)

\begin{abstract}
A numerical approach called Fourier direct method (FDM) is applied to nonlinear propagation of optical pulses with the central wavelength $800 \mathrm{~nm}$, the width $2.67-12.00 \mathrm{fs}$, and the peak power $25-6870 \mathrm{~kW}$ in a fused-silica fiber. Bidirectional propagation, delayed Raman response, nonlinear dispersion (self-steepening, core dispersion), as well as correct linear dispersion are incorporated into "bidirectional propagation equations" which are derived directly from Maxwell's equations. These equations are solved for forward and backward waves, instead of the electric-field envelope as in the nonlinear Schrödinger equation (NLSE). They are integrated as multidimensional simultaneous evolution equations evolved in space. We investigate, both theoretically and numerically, the validity and the limitation of assumptions and approximations used for deriving the NLSE. Also, the accuracy and the efficiency of the FDM are compared quantitatively with those of the finite-difference time-domain numerical approach. The time-domain size $500 \mathrm{fs}$ and the number of grid points in time 2048 are chosen to investigate numerically intensity spectra, spectral phases, and temporal electric-field profiles up to the propagation distance $1.0 \mathrm{~mm}$. On the intensity spectrum of a few-optical-cycle pulses, the self-steepening, core dispersion, and the delayed Raman response appear as dominant, middle, and slight effects, respectively. The delayed Raman response and the core dispersion reduce the effective nonlinearity. Correct linear dispersion is important since it affects the intensity spectrum sensitively. For the compression of femtosecond optical pulses by the complete phase compensation, the shortness and the pulse quality of compressed pulses are remarkably improved by the intense initial peak power rather than by the short initial pulse width or by the propagation distance longer than $0.1 \mathrm{~mm}$. They will be compressed as short as $0.3 \mathrm{fs}$ below the damage threshold of fused-silica fiber $6 \mathrm{MW}$. It is demonstrated that the carrier envelope phase (CEP) causes the difference on the temporal electric-field profile and the intensity spectrum for the initial peak power of the order of megawatts. At the propagation distance longer than the coherence length for third-order harmonics, the difference grows in the spectral components around the third-order and higher-order harmonics. The CEP can be a sensitive marker to monitor the evolution of nonlinear optical process by a few-optical-cycle electric-field wave-packet source.
\end{abstract}

DOI: 10.1103/PhysRevA.72.063802 PACS number(s): 42.50.Nn, 42.65. - k, 42.65.Re, 42.81.-i

\section{INTRODUCTION}

For recent remarkable development in few-cycle photonics, exact and practical analysis on extremely short optical pulses propagating in nonlinear fibers is quite important. Elucidation of complex nonlinear optical propagation in recently developed group-delay-dispersion (GDD) controlled fibersphotonic crystal fibers, tapered glass fibers, and so onfacilitating ultrabroadband coherent light generation in low power is an urgent problem. Correct and practical computational methods are necessary for the analysis of few-cycle and/or ultrabroadband optical pulses with the carrier envelope phase (CEP) effect, shorter than $2.8 \mathrm{fs}$ which we have realized recently in the visible region [1-3].

For the analysis of nonlinear propagation of optical pulses, the nonlinear Schrödinger equation (NLSE) for the electric-field envelope has been used conventionally as well as its modifications. The NLSE, composed of only the lowest-order linear dispersion and the nonlinearity, originates in Maxwell's equations but is derived on assumptions or approximations such as (1) the unidirectional propagation ap-

\footnotetext{
*Electronic address: yomizuta@eng.hokudai.ac.jp
}

proximation, (2) the restricted frequency range, (3) the instantaneous or frequency-independent nonlinear response, (4) the quadratic linear dispersion, and (5) the slowlyvarying-envelope approximation (SVEA) [4,5]. However, the optical pulses considered in this paper have the width as short as 2.67 fs and the peak power as large as 6.8 $\times 10^{3} \mathrm{~kW}$. Then, the spectrum as wide as the carrier frequency is broadened furthermore by nonlinear effects during pulse propagation. In addition, separating the envelope from the temporal electric-field profile becomes meaningless. Thus, it is required to treat electromagnetic waves under large nonlinear and dispersion effects rigorously which are neglected in the NLSE.

To cope with this situation, there have been many works on the realistic linear dispersion [6-8], the delayed Raman response $[9,10]$, and the core dispersion $[11,12]$. These effects are necessarily considered in all for realistic analysis, however, the latter two were not considered, for example, in [8]. Sometimes, the SVEA on the assumption of the envelope varying slowly in space and time is felt too strict, and is relaxed to the SEWA under the assumption where the group velocity and the phase velocity are close [5]. Recently, the simultaneous evolution equations such as the onedimensionalized Maxwell's equations are solved by the 
finite-difference time-domain numerical approach (FDTD method) [13-16].

In this paper, we introduce a method called "Fourier direct method (FDM)," which originated in the split-step Fourier method usually employed to solve the NLSE [17-20]. The FDM has been extended so as to treat multidimensional evolution equations or general nonlinear terms including differentials or integrals. The FDM is based on "bidirectional propagation equations" for forward and backward waves, instead of the electric-field envelope in the NLSE. They are derived, without approximations, from Maxwell's equations averaged over a cross section normal to the fiber in Sec. II B. They include all realistic linear and nonlinear effects: correct linear dispersion, the delayed Raman response, and nonlinear dispersions (self-steepening, core dispersion). As for the nonlinear polarization, it is assumed to be third order in the electric field in the nonresonant frequency range. However, the nonlinear polarization itself is not supposed to be small in contrast to [9] where the unidirectional propagation equation is derived by the formal Taylor expansion. It is indispensable to clarify systematically and quantitatively the relation with and the difference from other methods used conventionally. For this purpose, we rederive the NLSE straightforwardly from one of the bidirectional propagation equations without the SVEA in Sec. II E, in contrast to the conventional derivation.

The bidirectional propagation equations are multidimensional simultaneous evolution equations. They are integrated in space for the linear term and the nonlinear term alternatively like the split-step Fourier method (Sec. III). This FDM is stable and accurate for long propagation distance under the wide range of analytic parameter values. It is as efficient as the FDTD method to obtain the same accuracy. The temporal electric-field profile and the intensity spectrum are obtained at each spatial position. Thus, they are directly compared with experimental results free from additional data processing. In addition, the cause of numerical troubles can be traced easily.

We deal with the present FDM, developed for the nonlinear propagation of femtosecond optical pulses in fused-silica fibers, as follows.

(1) We clarify the relation between the bidirectional propagation equations and the NLSE, to comprehend systematically and quantitatively the validity and the limitation of the assumptions and approximations used in conventional theoretical methods (Sec. II E).

(2) We compare the results calculated by the FDM and the NLSE, and clarify differences of the effects caused by the above-mentioned assumptions and approximations (Sec. IV).

(3) We point out a possibility of subfemtosecond pulse compression using a conventional silica fiber under the practical condition (Sec. V B).

(4) We demonstrate the effects generated by the CEP on third-order and higher-order harmonic spectral components of few-optical-cycle pulses in low power (Sec. VI).

\section{FOURIER DIRECT METHOD}

\section{A. Averaged Maxwell's equation}

In order to describe electromagnetic fields propagating in fibers, we start with

$$
\begin{gathered}
\nabla \times \mathbf{E}=-\frac{\partial \mathbf{B}}{\partial t}, \\
\nabla \times \mathbf{H}=\frac{\partial \mathbf{D}}{\partial t}+\mathbf{J},
\end{gathered}
$$

in Maxwell's equations as well as $\nabla \cdot \mathbf{D}=\rho$ and $\nabla \cdot \mathbf{B}=0$, where $\mathbf{E}$ and $\mathbf{H}$ are electric and magnetic fields, respectively, and $\mathbf{D}$ and $\mathbf{B}$ are electric and magnetic flux densities. Due to the absence of free charges in optical fibers, the charge density $\rho$ and the electric current density $\mathbf{J}$ vanish. Among the vacuum permittivity $\varepsilon_{0}$, vacuum permeability $\mu_{0}$, and the velocity of light in vacuum, $\varepsilon_{0} \mu_{0}=1 / c^{2}$ holds. Furthermore, $\mathbf{B}=\mu_{0} \mathbf{H}$ in nonmagnetic optical fibers.

Polarization-preserving fibers maintain the linear polarization of the light launched with the polarization along one of the principal axes of the fiber. We choose here the $z$ direction parallel to the fiber, the $x$ direction along the axis of polarization, and the $y$ direction normal to both the $x$ and $z$ directions. Since $\mathbf{E}$ and $\mathbf{H}$ have, in this case, only components $E_{x}$ and $H_{y}$, respectively, we derive a set of evolution equations as

$$
\begin{gathered}
\frac{\partial E}{\partial z}=-\frac{\partial B}{\partial t}, \\
-\frac{\partial H}{\partial z}=\frac{\partial D}{\partial t},
\end{gathered}
$$

after averaging Eq. (1) spatially over a cross section normal to the fiber on the assumption that the fields vanish at infinity. When this average [defined in (5) hereafter] is shown by $\langle\cdots\rangle_{\mathrm{av}}$, the quantities in (2) are defined as

$$
\begin{gathered}
E(z, t) \equiv\left\langle E_{x}(x, y, z, t)\right\rangle_{\mathrm{av}} \\
H(z, t) \equiv\left\langle H_{y}(x, y, z, t)\right\rangle_{\mathrm{av}} \\
B(z, t) \equiv\left\langle B_{y}(x, y, z, t)\right\rangle_{\mathrm{av}}=\mu_{0} H(z, t) \\
D(z, t) \equiv\left\langle D_{x}(x, y, z, t)\right\rangle_{\mathrm{av}}=\varepsilon_{0} E(z, t)+P_{\mathrm{L}}(z, t)+P_{\mathrm{N}}(z, t)
\end{gathered}
$$

where $P_{\mathrm{L}}(z, t)$ and $P_{\mathrm{N}}(z, t)$ are linear and nonlinear parts of the induced polarization, respectively, which depends on $E(z, t)$. Equation (2) is often solved by the FDTD method as evolved in time. However, time-dependent results at fixed positions are more favorable to experiments, and several dispersion effects are incorporated properly in the frequency domain. Thus, we consider Eq. (2) in the frequency domain, as evolved in space.

In the frequency domain, the Fourier transform of the electric field $E_{x}(x, y, z, t)$ is shown as $F(x, y, \omega) \widetilde{E}(z, \omega)$, to separate the dependence on the transverse coordinates by $F(x, y, \omega)$. We stress here the importance of the frequencydependence of this function to discuss the core dispersion, the change of the effective core area according to the frequency of the field. If we denote the Fourier transform in time by $\mathrm{F}$, 


$$
\mathrm{F} E(z, t)=\int_{-\infty}^{\infty} d t e^{i \omega t} E(z, t)=\langle F(x, y, \omega) \widetilde{E}(z, \omega)\rangle_{\mathrm{av}}=\tilde{E}(z, \omega),
$$

where the average is actually defined as

$$
\begin{gathered}
\langle\cdots\rangle_{\mathrm{av}} \equiv \frac{1}{A^{(2)}(\omega)} \iint d x d y F(x, y, \omega) \cdots, \\
A^{(n)}(\omega) \equiv \iint d x d y|F(x, y, \omega)|^{n} .
\end{gathered}
$$

Equations in (2) are Fourier-transformed in time as

$$
\begin{gathered}
\frac{\partial \widetilde{E}}{\partial z}=-\frac{i \omega}{c} \widetilde{H}, \\
\frac{\partial \tilde{H}}{\partial z}=-\frac{i \omega}{c}\left(n_{\mathrm{L}}^{2} \widetilde{E}+\mathrm{F} \frac{P_{\mathrm{N}}}{\varepsilon_{0}}\right),
\end{gathered}
$$

to be solved for $\widetilde{E}(z, \omega)=\mathrm{F} E(z, t)$ and $\tilde{H}(z, \omega)=-c$ $\mu_{0} \mathrm{FH}(z, t)$. The term including the linear refractive index $n_{\mathrm{L}}(\omega)$ in Eq. (6) comes from the flux density in Eq. (2) as

$$
\begin{aligned}
\mathrm{F} \frac{D}{\varepsilon_{0}} & =\mathrm{F}\left(E+\frac{P_{\mathrm{L}}}{\varepsilon_{0}}+\frac{P_{\mathrm{N}}}{\varepsilon_{0}}\right) \\
& =\mathrm{F}\left[E(z, t)+\int_{-\infty}^{t} d t_{1} \chi^{(1)}\left(t-t_{1}\right) E\left(z, t_{1}\right)+\frac{P_{\mathrm{N}}}{\varepsilon_{0}}\right] \\
& =\left[n_{\mathrm{L}}(\omega)\right]^{2} \widetilde{E}+\mathrm{F} \frac{P_{\mathrm{N}}}{\varepsilon_{0}},
\end{aligned}
$$

where $n_{\mathrm{L}}(\omega)=n_{\mathrm{L}}^{\mathrm{R}}(\omega)+i n_{\mathrm{L}}^{\mathrm{I}}(\omega)$ is related to the linear dielectric constant $\varepsilon_{\mathrm{L}}(\omega)$ and the linear electric susceptibility $\tilde{\chi}^{(1)}(\omega)$ $=\mathrm{F} \chi^{(1)}(t)$, the Fourier-transformed linear response function $\chi^{(1)}(t)$, as $\left[n_{\mathrm{L}}(\omega)\right]^{2}=\varepsilon_{\mathrm{L}}(\omega)=1+\tilde{\chi}^{(1)}(\omega)$. This complex and frequency-dependent refractive index includes the effects of absorption and finite-time response at the linear stage, assuming the medium response isotropic and local. For the nonlinear induced polarization, on the other hand, we use the following expression which is third order in $E_{x}(x, y, z, t)$ or $E(z, t)$, and includes the delayed Raman response represented by $\chi^{(3)} r(t)$ :

$$
\mathrm{F} \frac{P_{\mathrm{N}}(z, t)}{\varepsilon_{0}}=\mathrm{F}\left\langle\chi^{(3)} E_{x}(x, y, z, t) \int_{-\infty}^{t} d t_{1} r\left(t-t_{1}\right) E_{x}^{2}\left(x, y, z, t_{1}\right)\right\rangle \text { av }
$$

\section{B. Bidirectional propagation equation}

For successful analysis of high-power and ultrashort optical pulses propagating in a fiber, we need to scale the spatial coordinate, the time, the frequency, and the fields in Eq. (6) by the characteristic length $Z$, the characteristic time $T$, and the peak power of the pulse $P$ as follows:

$$
\xi=z / Z,
$$

$$
\tau=(t-z / v) / T,
$$

$$
\omega_{\mathrm{C}}=T \omega,
$$

$$
\left(\begin{array}{c}
\widetilde{E}(z, \omega) \\
\widetilde{H}(z, \omega)
\end{array}\right)=E_{\mathrm{p}}(\omega)\left(\begin{array}{l}
B_{1}\left(\xi, \omega_{\mathrm{C}}\right) \\
B_{2}\left(\xi, \omega_{\mathrm{C}}\right)
\end{array}\right),
$$

where $v$ is the velocity of the coordinate system moving together with the pulse. As $T, Z$, and $v$, at present, we properly choose the half-width of the pulse at $1 / e$-intensity point $\left[t_{1} / 1.665\right.$ for the Gaussian profile where $t_{1}$ is its full width at half maximum (FWHM)], the dispersion length $L_{\mathrm{LD}}$ [defined below in (29a)] and the group velocity, respectively. We scale electric and magnetic fields by $E_{\mathrm{p}}(\omega)$ $\equiv \sqrt{P /\left[c \varepsilon_{0} n_{\mathrm{L}}^{\mathrm{R}}(\omega) A^{(2)}(\omega)\right]}$ referring to

$$
P=\max _{t}\left\{c \varepsilon_{0} n_{0} \iint d x d y|E(x, y, z, t)|^{2}\right\},
$$

where $\max _{t}\{\cdots\}$ means the maximum in time, and $n_{0}$ $\equiv n_{\mathrm{L}}^{\mathrm{R}}\left(\omega_{0}\right)$ at the central angular frequency $\omega_{0}$. Then, $\max _{\tau}\left\{\left|\mathrm{F}^{-1} B_{1}\left(\xi, \omega_{\mathrm{C}}\right)\right|^{2}\right\}$ is $O(1)$, and Eq. (6) reduces to

$$
\begin{gathered}
\frac{\partial B_{1}}{\partial \xi}=-i \omega Z\left(\frac{B_{1}}{v}+\frac{B_{2}}{c}\right), \\
\frac{\partial B_{2}}{\partial \xi}=-i \omega Z\left(\frac{B_{2}}{v}+\frac{n_{\mathrm{L}}^{2} B_{1}}{c}\right)+G_{\mathrm{N}}, \\
G_{\mathrm{N}} \equiv \frac{Z}{E_{\mathrm{p}}(\omega)}\left(\frac{-i \omega}{c} \mathrm{~F} \frac{P_{\mathrm{N}}}{\varepsilon_{0}}\right) .
\end{gathered}
$$

Here we define $A_{1,2}$ from $B_{1,2}$, and express $B_{1,2}$ by $A_{1,2}$ inversely as follows:

$$
\begin{gathered}
\mathbf{A}=\left(\begin{array}{l}
A_{1} \\
A_{2}
\end{array}\right)=\frac{1}{n_{\mathrm{L}}+n_{\mathrm{L}}^{*}}\left(\begin{array}{c}
n_{\mathrm{L}}^{*} B_{1}-B_{2} \\
n_{\mathrm{L}} B_{1}+B_{2}
\end{array}\right), \\
\mathbf{B}=\left(\begin{array}{l}
B_{1} \\
B_{2}
\end{array}\right)=\left(\begin{array}{c}
A_{1}+A_{2} \\
n_{\mathrm{L}}^{*} A_{2}-n_{\mathrm{L}} A_{1}
\end{array}\right) .
\end{gathered}
$$

Using these $A_{1,2}$, we diagonalize the linear terms in the right side of Eqs. (10a) and (10b) as

$$
\begin{gathered}
\frac{\partial A_{1}}{\partial \xi}=F_{\mathrm{L} 1}-F_{\mathrm{N}}, \quad F_{\mathrm{L} 1} \equiv i Z\left(\beta-\frac{\omega}{v}\right) A_{1}, \\
\frac{\partial A_{2}}{\partial \xi}=F_{\mathrm{L} 2}+F_{\mathrm{N}}, \quad F_{\mathrm{L} 2} \equiv i Z\left(-\beta^{*}-\frac{\omega}{v}\right) A_{2}, \\
F_{\mathrm{N}} \equiv \frac{G_{\mathrm{N}}}{n_{\mathrm{L}}+n_{\mathrm{L}}^{*}} \equiv \frac{Z}{2 n_{\mathrm{L}}^{\mathrm{R}}(\omega) E_{\mathrm{p}}(\omega)}\left(\frac{-i \omega}{c} \mathrm{~F} \frac{P_{\mathrm{N}}}{\varepsilon_{0}}\right), \\
\beta(\omega) \equiv \frac{\omega n_{\mathrm{L}}(\omega)}{c} .
\end{gathered}
$$

The refractive index $n_{\mathrm{L}}(\omega)=n_{\mathrm{L}}^{\mathrm{R}}(\omega)+i n_{\mathrm{L}}^{\mathrm{I}}(\omega)$, and the propagation constant $\beta(\omega)$ are extended to complex numbers here to 
express the linear absorption by their imaginary part. This extension modifies Eqs. (10a) and (10b) to

$$
\begin{gathered}
\frac{\partial B_{1}}{\partial \xi}=-i \omega Z\left(\frac{B_{1}}{v}+\frac{B_{2}}{c}\right), \\
\frac{\partial B_{2}}{\partial \xi}=-i \omega Z\left(\frac{B_{2}}{v}+\frac{\left|n_{\mathrm{L}}\right|^{2} B_{1}}{c}\right)+G_{\mathrm{N}}-\frac{2 n_{\mathrm{L}}^{\mathrm{I}} \omega Z}{c} B_{2}
\end{gathered}
$$

with a new term proportional to $n_{\mathrm{L}}^{\mathrm{I}}$ causing the expense of energy.

Without the nonlinear term $F_{\mathrm{N}}$, we integrate Eq. (12a) in $\xi$ (accordingly $z$ ) for $A_{1,2}$, and their inverse Fourier transforms

$$
\left(\begin{array}{l}
a_{1} \\
a_{2}
\end{array}\right)=\mathrm{F}^{-1}\left(\begin{array}{l}
A_{1} \\
A_{2}
\end{array}\right)=\frac{1}{2 \pi} \int_{-\infty}^{\infty} d t\left(\begin{array}{c}
G_{+}(\omega) e^{i\{(\beta-\omega / v) z-\omega t\}} \\
G_{-}(\omega) e^{i\left\{\left(-\beta^{*}-\omega / v\right) z-\omega t\right\}}
\end{array}\right)
$$

show that $a_{1}$ and $a_{2}$ are the right-traveling forward wave and the left-traveling backward wave, respectively. Thus, we see from Eq. (11a) that the initial condition of only the forward wave is given by $A_{1}=B_{1}$ and $A_{2}=0$ that leads $\tilde{H}(z, \omega)$ $=-n_{\mathrm{L}}(\omega) \widetilde{E}(z, \omega)$ at $z=0$ from Eq. (9d). In addition, Eq. (14) shows that each of $a_{1}$ and $a_{2}$ attenuates with $z$, since $\operatorname{Im} \beta(\omega)>0$.

We find that Eq. (12) describes even such a situation that two extremely short pulses close to an optical cycle propagate in opposite directions, collide with a strong interaction, and leave far away from each other. Even if so, the term $F_{\mathrm{N}}$ describes not only the nonlinear self-interaction but also the nonlinear interaction between two pulses during the collision. Therefore, we call Eq. (12a) "bidirectional propagation equations." This approach is more natural and general for analyzing optical pulses in fibers, compared with [9] where formal factorization and the Taylor expansion were employed.

The following equations derived from Eq. (12) describe changes in spectral intensities of the forward wave, backward wave, and the total wave along with propagation:

$$
\begin{gathered}
\frac{\partial\left(A_{1} A_{1}^{*}\right)}{\partial \xi}=-\alpha Z A_{1} A_{1}^{*}-2 \operatorname{Re} A_{1}^{*} F_{\mathrm{N}}, \\
\frac{\partial\left(A_{2} A_{2}^{*}\right)}{\partial \xi}=-\alpha Z A_{2} A_{2}^{*}+2 \operatorname{Re} A_{2}^{*} F_{\mathrm{N}}, \\
\frac{\partial\left|A_{1}+A_{2}\right|^{2}}{\partial \xi}=-\alpha Z\left|A_{1}+A_{2}\right|^{2} .
\end{gathered}
$$

In addition to the linear attenuation shown by $\alpha \equiv 2 \omega n_{\mathrm{L}}^{\mathrm{I}} / c$, intensities of the forward wave and the backward wave are changed by $-2 \operatorname{Re} A_{1}^{*} F_{\mathrm{N}}$ and $2 \operatorname{Re} A_{2}^{*} F_{\mathrm{N}}$, respectively, including both the self-interaction and the interaction between forward and backward waves, as (15a) and (15b) show. By contrast, $(15 \mathrm{c})$ shows that the change in the intensity of the total wave is irrespective of the nonlinear interaction. These consequences are useful for checking the calculated results.

\section{Linear term}

Considering isotropic propagation accompanied by the attenuation in both directions in a silica fiber, we adopt the following modified Sellmeier equation for the linear refractive index [8]:

$$
\left[n_{\mathrm{L}}(\omega)\right]^{2}=1+\sum_{j=1}^{N} S_{j}\left(\frac{1}{\omega_{j}-i \Gamma_{j}-\omega}+\frac{1}{\omega_{j}+i \Gamma_{j}+\omega}\right),
$$

where $N=2$, and $\omega_{1}=0.21 \mathrm{rad} / \mathrm{fs}, S_{1}=0.06 \mathrm{fs}^{-1}, \Gamma_{1}=3.77$ $\times 10^{-3} \mathrm{rad} / \mathrm{fs}, \quad \omega_{2}=18.9 \mathrm{rad} / \mathrm{fs}, \quad S_{2}=10.2 \mathrm{fs}^{-1}, \quad \Gamma_{2}=0.00$ $\mathrm{rad} / \mathrm{fs}$.

This $n_{\mathrm{L}}(\omega)$ shows $n_{\mathrm{L}}^{*}(-\omega)=n_{\mathrm{L}}(\omega)$, or $n_{\mathrm{L}}^{\mathrm{R}}(-\omega)=n_{\mathrm{L}}^{\mathrm{R}}(\omega)$, $n_{\mathrm{L}}^{\mathrm{I}}(-\omega)=-n_{\mathrm{L}}^{\mathrm{I}}(\omega)$, and $\omega n_{\mathrm{L}}^{\mathrm{I}}(\omega) / c=\operatorname{Im} \beta(\omega)>0$ as long as $n_{\mathrm{L}}^{\mathrm{R}}(\omega)>0$. At the wavelength $\lambda_{0}=800 \mathrm{~nm}$ (the angular frequency is $\left.\omega_{0}=2.356 \mathrm{rad} / \mathrm{fs}\right)$, (16) gives $n_{\mathrm{L}}^{\mathrm{R}}\left(\omega_{0}\right)=1.446$.

\section{Nonlinear term}

From Eqs. (8) and (12b), the nonlinear term in the bidirectional propagation equations $(12 \mathrm{a})$ is

$$
\begin{gathered}
F_{\mathrm{N}}=\frac{-i \omega Z}{c} \frac{\chi^{(3)}}{2 n_{\mathrm{L}}^{\mathrm{R}}(\omega) E_{\mathrm{p}}(\omega)} S_{\mathrm{B}}(\omega), \\
S_{\mathrm{B}}(\omega) \equiv\left\langle\mathrm{F} E_{x}(x, y, z, t) \int_{-\infty}^{t} d t_{1} r\left(t-t_{1}\right)\left[E_{x}\left(x, y, z, t_{1}\right)\right]^{2}\right\rangle_{\mathrm{av}} \\
=\left\langle\mathrm{F} E_{x}(x, y, z, t) \mathrm{F}^{-1} \widetilde{R}(\omega) \mathrm{F}\left[E_{x}(x, y, z, t)\right]^{2}\right\rangle_{\mathrm{av}}
\end{gathered}
$$

by the convolution theorem, where $\mathrm{F}_{\text {or }} \mathrm{F}^{-1}$ operate on all on the right of them, and $E_{x}(x, y, z, t)$ is expressed by the scaled electric field $b_{1}(\xi, \tau) \equiv \mathrm{F}^{-1} B_{1}\left(\xi, \omega_{\mathrm{C}}\right)$ from Eq. (9d) as

$$
E_{x}(x, y, z, t)=\mathrm{F}^{-1} F(x, y, \omega) E_{\mathrm{p}}(\omega) \mathrm{F} b_{1}(\xi, \tau) .
$$

Furthermore, the nonlinear response function which is composed of the instantaneous part and the delayed Raman part, and its Fourier transform are [9]

$$
\begin{gathered}
r(t)=\left(1-f_{\mathrm{R}}\right) \delta(t)+f_{\mathrm{R}} h_{\mathrm{R}}(t) \\
\widetilde{R}(\omega)=\mathrm{F} r(t)=\left(1-f_{\mathrm{R}}\right)+f_{\mathrm{R}} \mathrm{F} h_{\mathrm{R}}(t) \\
\mathrm{F} h_{\mathrm{R}}(t)=\tilde{H}\left(\omega-\omega_{0}\right)+\widetilde{H}^{*}\left(-\omega-\omega_{0}\right) \\
\tilde{H}(\omega)=\mathrm{F} \frac{\tau_{1}^{2}+\tau_{2}^{2}}{\tau_{1} \tau_{2}^{2}} e^{-t / \tau_{2}} \sin \left(\frac{t}{\tau_{1}}\right)=\frac{\tau_{1}^{2}+\tau_{2}^{2}}{\tau_{1}^{2}\left(1-i \omega \tau_{2}\right)^{2}+\tau_{2}^{2}}
\end{gathered}
$$

If we define $\omega_{\mathrm{S}, \mathrm{A}}$ as the frequency of the minimum and the maximum of $\operatorname{Im} \widetilde{H}\left(\omega-\omega_{0}\right)$, and $\delta \equiv\left(\tau_{2} / \tau_{1}\right)^{2}$,

$$
\left|\omega_{\mathrm{S}, \mathrm{A}}-\omega_{0}\right|=\frac{1}{\tau_{2}} \sqrt{\frac{(\delta-1)+2 \sqrt{\delta^{2}+\delta+1}}{3}} .
$$

In the following we define $S_{\mathrm{A}}(\omega)$ which is closely related to $S_{\mathrm{B}}(\omega)$ in $(17 \mathrm{~b})$. In addition, we define $\Delta(\omega)$ for measuring the difference between $S_{\mathrm{B}}(\omega)$ and $S_{\mathrm{A}}(\omega)$ : 


$$
S_{\mathrm{A}}(\omega) \equiv\left\langle\left[F(x, y, \omega) E_{\mathrm{p}}(\omega)\right]^{3} \mathrm{~F} b_{1}(\xi, \tau) \mathrm{F}^{-1} \widetilde{R}(\omega) \mathrm{F}\left[b_{1}(\xi, \tau)\right]^{2}\right\rangle_{\mathrm{av}}
$$

$$
\begin{gathered}
=\frac{A^{(4)}(\omega)}{A^{(2)}(\omega)}\left[E_{\mathrm{p}}(\omega)\right]^{3} \mathrm{~F} b_{1}(\xi, \tau) \mathrm{F}^{-1} \widetilde{R}(\omega) \mathrm{F}\left[b_{1}(\xi, \tau)\right]^{2}, \\
\Delta(\omega) \equiv S_{\mathrm{B}}(\omega) / S_{\mathrm{A}}(\omega)-1 .
\end{gathered}
$$

In $S_{\mathrm{A}}(\omega)$, we average $[F(x, y, \omega)]^{3}$ collected from $E_{x}(x, y, z, t)$ 's as (18) over the cross section of fiber in advance of $\mathrm{F}$ or $\mathrm{F}^{-1}$, to be $A^{(4)}(\omega) / A^{(2)}(\omega)$. Then, $F_{\mathrm{N}}$ is rewritten further as:

$$
\begin{gathered}
F_{\mathrm{N}}=i \gamma_{\mathrm{FDM}}(\omega) \mathrm{F} b_{1}(\xi, \tau) \mathrm{F}^{-1} \tilde{R}(\omega) \mathrm{F}\left[b_{1}(\xi, \tau)\right]^{2}, \\
\gamma_{\mathrm{FDM}}(\omega) \equiv \frac{\omega Z}{c} \frac{\chi^{(3)}}{2 n_{\mathrm{L}}^{\mathrm{R}}(\omega)}\left[\tilde{E}_{\mathrm{p}}(\omega)\right]^{2} \\
{\left[\tilde{E}_{\mathrm{p}}(\omega)\right]^{2} \equiv \frac{P}{c \varepsilon_{0} n_{\mathrm{L}}^{\mathrm{R}}(\omega) A_{\mathrm{eff}}(\omega)}} \\
A_{\mathrm{eff}}(\omega) \equiv \frac{\left[A^{(2)}(\omega)\right]^{2}}{A^{(4)}(\omega)[1+\Delta(\omega)]}
\end{gathered}
$$

where $A_{\text {eff }}(\omega)$ is the effective core area. Equation (22a) is the form of $F_{\mathrm{N}}$ actually used for the present method instead of Eq. (12b).

Equation (22b) allows us to consider the frequency dependence of the coefficient of the nonlinear term $\gamma_{\mathrm{FDM}}(\omega)$ which causes the "nonlinear dispersion" leading to such effects as the asymmetry or self-steepening in the pulse. Instead of Eq. (22b), we can evaluate $\gamma_{\mathrm{FDM}}(\omega)$ as

$$
\gamma_{\mathrm{FDM}}(\omega)=\gamma_{\mathrm{FDM}}\left(\omega_{0}\right) \frac{\omega}{\omega_{0}}\left[\frac{n_{\mathrm{L}}^{\mathrm{R}}(\omega)}{n_{\mathrm{L}}^{\mathrm{R}}\left(\omega_{0}\right)}\right]^{-2}\left[\frac{A_{\mathrm{eff}}(\omega)}{A_{\mathrm{eff}}\left(\omega_{0}\right)}\right]^{-1}
$$

from the value of $\gamma_{\mathrm{FDM}}(\omega)$ at the central frequency $\omega_{0}$, and the behavior of $n_{\mathrm{L}}^{\mathrm{R}}(\omega)$ and $A_{\text {eff }}(\omega)$ around this $\omega_{0}$. For the electric field in single-mode fibers, its intensity distribution in a cross section of the fiber is approximated as axial-symmetric Gaussian, and $F(x, y, \omega)$ $=\exp \left\{-\left(x^{2}+y^{2}\right) /[w(\omega)]^{2}\right\}$ is used with the frequencydependent effective core radius $w(\omega)$. On the other hand, when $F(x, y, \omega) E_{\mathrm{p}}(\omega)$ depends on $\omega$ weakly compared with $B_{1}\left(\xi, \omega_{\mathrm{C}}\right)$, then $E_{x}(x, y, z, t) \simeq F(x, y) E_{\mathrm{p}} b_{1}(\xi, \tau)$ from Eq. (18), $S_{\mathrm{B}}(\omega) \simeq S_{\mathrm{A}}(\omega)$, and $\Delta(\omega)$ almost vanishes even for pulses with wide spectra. As a result, $A_{\mathrm{eff}}(\omega) \simeq \pi[w(\omega)]^{2}$, and $A_{\text {eff }}(\omega) / A_{\text {eff }}\left(\omega_{0}\right)$ is estimated as $\left[w(\omega) / w\left(\omega_{0}\right)\right]^{2}$.

The value of the nonlinear susceptibility is obtained from

$$
\begin{aligned}
n_{2}= & \left(3 \chi^{(3)}\right) /\left[8 n_{\mathrm{L}}^{\mathrm{R}}\left(\omega_{0}\right)\right]=1.220 \times 10^{-22}(\mathrm{~V} / \mathrm{m})^{-2} \\
& {\left[=40 \pi \varepsilon_{0} n_{2}(\mathrm{esu}), n_{2}(\mathrm{esu})=1.097 \times 10^{-13} \mathrm{esu}\right] }
\end{aligned}
$$

for the fused-silica fiber [21]. In addition, $\tau_{1}=12.2 \mathrm{fs}, \tau_{2}$ $=32.0 \mathrm{fs}$ and $f_{\mathrm{R}}=0.30$ are used in the nonlinear response function $r(t)$ referring to [9]. By these values, $\omega_{\mathrm{S}}$ $=2.274 \mathrm{rad} /$ fs and $\omega_{\mathrm{A}}=2.438 \mathrm{rad} /$ fs from Eq. (20).

\section{E. Relation with the nonlinear Schrödinger equation}

When the pulse spectrum localizes around the central frequency, we can separate clearly the envelope wave $a_{ \pm}(\xi, \tau)$ from each of the forward wave $a_{1}(\xi, \tau)$ and the backward wave $a_{2}(\xi, \tau)$ as

$$
a_{1}(\xi, \tau)=\left[a_{+}(\xi, \tau) e^{i\left(\beta_{0 \mathrm{C}} \xi-\omega_{0 \mathrm{C}} \tau\right)}+a_{+}^{*}(\xi, \tau) e^{i\left(-\beta_{0 \mathrm{C}}+\omega_{0 \mathrm{C}} \tau\right)}\right] / 2,
$$

$$
a_{2}(\xi, \tau)=\left[a_{-}(\xi, \tau) e^{i\left(\beta_{0 \mathrm{C}} \xi+\omega_{0 \mathrm{C}} \tau\right)}+a_{-}^{*}(\xi, \tau) e^{i\left(-\beta_{0 \mathrm{C}} \xi-\omega_{0 \mathrm{C}} \tau\right)}\right] / 2,
$$

where $\omega_{0 \mathrm{C}}=T \omega_{0}$ and $\beta_{0 \mathrm{C}}=Z \beta\left(\omega_{0}\right)=Z \beta_{0}$. They are Fourier transformed as

$$
\begin{aligned}
A_{1}\left(\xi, \omega_{\mathrm{C}}\right)= & {\left[A_{+}\left(\xi, \omega_{\mathrm{C}}-\omega_{0 \mathrm{C}}\right) e^{i \beta_{0 \mathrm{C}} \xi}\right.} \\
& \left.+A_{+}^{*}\left(\xi,-\omega_{\mathrm{C}}-\omega_{0 \mathrm{C}}\right) e^{-i \beta_{0 \mathrm{C}} \xi}\right] / 2, \\
A_{2}\left(\xi, \omega_{\mathrm{C}}\right)= & {\left[A_{-}\left(\xi, \omega_{\mathrm{C}}+\omega_{0 \mathrm{C}}\right) e^{i \beta_{0 \mathrm{C}} \xi}\right.} \\
& \left.+A_{-}^{*}\left(\xi,-\omega_{\mathrm{C}}+\omega_{0 \mathrm{C}}\right) e^{-i \beta_{0 \mathrm{C}} \xi}\right] / 2,
\end{aligned}
$$

where

$$
\begin{aligned}
& A_{ \pm}\left(\xi, \omega_{\mathrm{C}} \mp \omega_{0 \mathrm{C}}\right)=\mathrm{F} a_{ \pm}(\xi, \tau) e^{\mp i \omega_{0 \mathrm{C}} \tau}, \\
& A_{ \pm}^{*}\left(\xi,-\omega_{\mathrm{C}} \mp \omega_{0 \mathrm{C}}\right)=\mathrm{F} a_{ \pm}^{*}(\xi, \tau) e^{ \pm i \omega_{0 \mathrm{C}} \tau} .
\end{aligned}
$$

For simplicity, we define here the "envelope-wave transform operator" $\mathrm{E}$ to take out the envelope wave from the forward wave as $\mathrm{E} a_{1}(\xi, \tau)=a_{+}(\xi, \tau), \quad \mathrm{E} A_{1}\left(\xi, \omega_{\mathrm{C}}\right)=A_{+}\left(\xi, \omega_{\mathrm{C}}\right)$, and $\mathrm{E}\left(\partial A_{1} / \partial \xi\right)=\partial A_{+} / \partial \xi+i \beta_{0 \mathrm{C}} A_{+}$.

Hereafter, we derive the NLSE for $a_{+}(\xi, \tau)$ which is composed of the lowest-order linear dispersion and nonlinearity. We begin with only the first of (12a) for the forward wave with $\mathrm{EF}^{-1}$ operated on both sides:

$$
\mathrm{EF}^{-1} \frac{\partial A_{1}}{\partial \xi}=\mathrm{EF}^{-1}\left(F_{\mathrm{L} 1}-F_{\mathrm{N}}\right)
$$

While deriving the NLSE, we use the following assumptions and approximations (see Appendix A for detail).

(1) The quadratic linear dispersion: $\beta(\omega)$ is expanded into the Taylor series around $\omega_{0}$, and $\beta(\omega)-\beta\left(\omega_{0}\right) \simeq \beta_{1}\left(\omega-\omega_{0}\right)$ $+\beta_{2}\left(\omega-\omega_{0}\right)^{2} / 2$, where $\beta_{n} \equiv \beta^{(n)}\left(\omega_{0}\right)$. This expansion is valid only when the spectrum of the pulse is localized around $\omega_{0}$.

(2) The restricted frequency range: The terms proportional to $e^{ \pm 3 i \omega_{0} t}$ are dropped. This approximation is valid as far as the spectrum of the pulse is confined sufficiently within the range $-2 \omega_{0}<\omega<2 \omega_{0}$.

(3) A nearly instantaneous nonlinear response: Only terms up to the first order in the Taylor series in $\omega-\omega_{0}$ of the Fourier transform of the nonlinear response function in $(19 \mathrm{~b})$ are left as $\widetilde{R}(\omega) \simeq 1+\widetilde{R}^{(1)}\left(\omega-\omega_{0}\right)$, where $\widetilde{R}^{(1)}$ $\equiv[d \widetilde{R}(\omega) / d \omega]_{\omega=\omega_{0}}$.

(4) A weak nonlinear dispersion such as the selfsteepening or the core dispersion: Only terms up to the first order in the Taylor series in $\omega-\omega_{0}$ of the coefficient of the 
nonlinear term $(22 \mathrm{~b})$ are left as $\gamma_{\mathrm{SPM}}(\omega) \equiv(3 / 4) \gamma_{\mathrm{FDM}}(\omega)$ $\simeq \gamma_{\mathrm{SPM}}^{(0)}+\gamma_{\mathrm{SPM}}^{(1)}\left(\omega-\omega_{0}\right)$, where $\gamma_{\mathrm{SPM}}^{(0)} \equiv \gamma_{\mathrm{SPM}}\left(\omega_{0}\right)$ and $\gamma_{\mathrm{SPM}}^{(1)}$ $\equiv\left[d \gamma_{\mathrm{SPM}}(\omega) / d \omega\right]_{\omega=\omega_{0}}$.

In addition,

(5) The unidirectional propagation approximation: Only the forward wave $A_{1}$ is considered throughout the propagation, and $B_{1} \simeq A_{1}, b_{1} \simeq a_{1}$. This approximation is used only in $F_{\mathrm{N}}$.

Then, we derive the following extended NLSE with the effect of the nonlinear dispersion and the Raman frequency shift.

$$
\begin{aligned}
\frac{\partial a_{+}}{\partial \xi}= & -\left(\beta_{1 \mathrm{C}}-\frac{Z / T}{v}\right) \frac{\partial a_{+}}{\partial \tau}-\frac{i \beta_{2 \mathrm{C}}}{2} \frac{\partial^{2} a_{+}}{\partial \tau^{2}}-\frac{i(Z / T) \omega_{0 \mathrm{C}}}{v} a_{+} \\
& +i\left(\gamma_{\mathrm{SPM}}^{(0)}+\frac{i \gamma_{\mathrm{SPM}}^{(1)}}{T} \frac{\partial}{\partial \tau}\right) a_{+}\left(1-\frac{T_{\mathrm{R}}}{T} \frac{\partial}{\partial \tau}\right)\left|a_{+}\right|^{2},
\end{aligned}
$$

where

$$
\begin{gathered}
\beta_{1 \mathrm{C}} \equiv \frac{Z \beta_{1}}{T}, \quad \beta_{2 \mathrm{C}} \equiv \frac{Z \beta_{2}}{T^{2}}=\frac{Z}{L_{\mathrm{LD}}}, \quad L_{\mathrm{LD}} \equiv \frac{T^{2}}{\beta_{2}}, \\
\gamma_{\mathrm{SPM}}^{(0)}=Z \frac{\omega_{0}}{c} \frac{3 \chi^{(3)}}{8 n_{0}}\left[\widetilde{E}_{\mathrm{p}}\left(\omega_{0}\right)\right]^{2}=\frac{Z}{L_{\mathrm{N}}} \\
L_{\mathrm{N}} \equiv\left[\frac{\omega_{0}}{c} \frac{3 \chi^{(3)}}{8 n_{0}} \frac{P}{c \varepsilon_{0} n_{0} A_{\mathrm{eff}}\left(\omega_{0}\right)}\right]^{-1}, \\
T_{\mathrm{R}} \equiv \frac{1}{i} \widetilde{R}^{(1)}=\int_{0}^{\infty} r(t) t d t=\frac{2 f_{\mathrm{R}} \tau_{1}^{2} \tau_{2}}{\tau_{1}^{2}+\tau_{2}^{2}} .
\end{gathered}
$$

Here we define the dispersion length $L_{\mathrm{LD}}$ and the nonlinear length $L_{\mathrm{N}}$. When $Z$ is chosen as $L_{\mathrm{LD}}, \gamma_{\mathrm{SPM}}^{(0)}=L_{\mathrm{LD}} / L_{\mathrm{N}}=N^{2}$ with the soliton order $N$. According to Eq. (23), $\gamma_{\mathrm{SPM}}^{(1)}$ is obtained alternatively as follows:

$$
\gamma_{\mathrm{SPM}}^{(1)}=\gamma_{\mathrm{SPM}}^{(0)}\left[\frac{1}{\omega_{0}}-2 \frac{w^{\prime}\left(\omega_{0}\right)}{w\left(\omega_{0}\right)}-2 \frac{n_{\mathrm{L}}^{\prime}\left(\omega_{0}\right)}{n_{0}}\right] .
$$

We obtain $T_{\mathrm{R}}$ as Eq. (29d) by using $r(t)$ in Eq. (19), and estimate $T_{\mathrm{R}}=2.44$ fs by the value of $\tau_{1}, \tau_{2}$, and $f_{\mathrm{R}}$ at the end of Sec. II D.

Under the conditions that (1) $v$ is adjusted to the group velocity $1 / \beta_{1}$, (2) the phase factor $e^{-i \omega_{0} \beta_{1} z}$ independent of $t$ is separated from $a_{+}$, and (3) terms with $\gamma_{\mathrm{SPM}}^{(1)}$ or $T_{\mathrm{R}}$ are dropped, Eq. (28) reduces to the usual NLSE:

$$
\frac{\partial a_{+}}{\partial \xi}=-\frac{i \beta_{2 \mathrm{C}}}{2} \frac{\partial^{2} a_{+}}{\partial \tau^{2}}+i \gamma_{\mathrm{SPM}}^{(0)}\left|a_{+}\right|^{2} a_{+} .
$$

We used Eq. (28) to check numerically the correctness of both the Fourier direct method and Eq. (28) by matching all conditions in the FDM with those of Eq. (28): only the forward wave for the initial condition; the quadratic linear dispersion; the frequency range confined within the basic frequency range for $F_{\mathrm{N}}$; and neither the nonlinear dispersion nor the delayed Raman response.

In the conventional derivation of the extended NLSE (28) or the usual NLSE (31), furthermore, the SVEA is always employed $[4,5,20]$. As discussed in Appendix A, the second- differential wave propagation equation is transformed under the assumption of infinitesimal $\beta-\beta_{0}$, with the second derivative of the envelope neglected. By contrast, the present derivation starting with the first-differential equation for the forward wave (27) uses the unidirectional propagation approximation instead of the SVEA. This derivation is much more straightforward with less restrictions in the range of validity.

\section{NUMERICAL PROCEDURE}

We have developed the present FDM from the split-step Fourier method [17-20] to solve multidimensional simultaneous evolution equations such as Eq. (12a). We describe them here in the vector form as $\partial \mathbf{A} / \partial \xi=\mathbf{F}_{\mathrm{L}}\left[\mathbf{A}\left(\omega_{\mathrm{C}}, \xi\right)\right]$ $+\mathbf{F}_{\mathrm{N}}\left[\mathbf{A}\left(\omega_{\mathrm{C}}, \xi\right)\right]$, where $\mathbf{F}_{\mathrm{L}}, \mathbf{F}_{\mathrm{N}}$, and $\mathbf{A}$ are the linear term vector, nonlinear term vector, and the unknown vector, respectively. They are quantities in the frequency domain obtained at each spatial point $\xi=n \Delta \xi$, where $n$ and $\Delta \xi$ are the step number and the spatial increment, respectively.

We obtain explicitly the unknown vector $\mathbf{A}_{n+1}$ at the step $n+1$ from the known vector $\mathbf{A}_{n}$ at the step $n$ as evolved in space. During $\Delta \xi$, we integrate $\mathbf{F}_{\mathrm{L}}$ by $\Delta \xi / 2$ twice before and after the integration of $\mathbf{F}_{\mathrm{N}}$ by $\Delta \xi$, as in the split-step Fourier method. Since the linear term is diagonalized as $\mathbf{F}_{\mathrm{L}}[\mathbf{A}]$ $=\mathrm{LA}$ in the frequency domain, we integrate $\mathbf{F}_{\mathrm{L}}$ in the exponential form which refines stability, accuracy, and efficiency of computation. However, we cannot diagonalize $\mathbf{F}_{\mathrm{N}}$ including differentials or integrals neither in the time domain nor in the frequency domain. It is integrated by the corrected Euler method (or by the Runge-Kutta method) which is more general but may be less stable. This is another difference of the FDM from the split-step Fourier method to solve the NLSE, where the nonlinear term is diagonal in the time domain, and it can be integrated in the exponential form. Thus, the procedures during $\Delta \xi$ explained above are summarized as follows:

$$
\begin{gathered}
\mathbf{A}^{(1)}=\exp (\mathrm{L} \Delta \xi / 2) \mathbf{A}_{n}, \\
\mathbf{F}^{(1)}=\mathbf{F}_{\mathrm{N}}\left[\mathbf{A}^{(1)}\right], \quad \widetilde{\mathbf{A}}=\mathbf{A}^{(1)}+\mathbf{F}^{(1)} \Delta \xi, \\
\mathbf{F}^{(2)}=\mathbf{F}_{\mathrm{N}}[\tilde{\mathbf{A}}], \quad \mathbf{A}^{(2)}=\mathbf{A}^{(1)}+\left\{\mathbf{F}^{(1)}+\mathbf{F}^{(2)}\right\}(\Delta \xi / 2), \\
\mathbf{A}_{n+1}=\exp (\mathrm{L} \Delta \xi / 2) \mathbf{A}^{(2)} .
\end{gathered}
$$

By the relation (11b), $\mathbf{A}\left(\omega_{\mathrm{C}}, \xi\right)$ obtained at the spatial point $\xi$ is changed to $\mathbf{B}\left(\omega_{\mathrm{C}}, \xi\right)$, whose components are $\widetilde{E}(z, \omega)$ and $\widetilde{H}(z, \omega)$ as shown in Eq. (9d). The intensity spectrum $I(\nu)$ $=|\widetilde{E}|^{2}$ is directly obtained from $\widetilde{E}$ where $\nu=\omega / 2 \pi=c / \lambda$. The phase continuation is used when the spectral phase $\phi(\nu)$ $=\arg \widetilde{E}$ is shown. For calculating the nonlinear term or at regular outputs of results, $\widetilde{E}(z, \omega)$ is inversely Fouriertransformed into the temporal electric-field profile $E(t)$. For this purpose, the fast Fourier transform (FFT) is employed, which saves a large amount of calculations. In addition, the general framework of the FDM facilitates solving the ex- 
TABLE I. List of all conditions and parameter values. Figs., figure number; Cs., case number (Sld, solid curve; Dsh, dashed curve; Dot, dotted curve); Mtd., method [FD, Fourier direct method; SG, nonlinear Schrödinger (SPM-GVD) equation]; Pro., propagation (1, unidirectional; 2, bidirectional); Rng., frequency range (B, basic; A, all); Nres., nonlinear response (I, instantaneous; D, delayed Raman); Ndis., nonlinear dispersion (S, self-steepening; F, flat core dispersion; T, theoretical core dispersion; E, experimental core dispersion); Ldis., linear dispersion (N, no dispersion; 2, second order; 3, third order; 4, fourth order; 5, fifth order; S, Sellmeier; $\mathrm{S}_{\mathrm{m}}$, modified Sellmeier with absorption); Ini., envelope and conditions for the initial temporal electric-field profile (G, Gaussian; S, sech; E, experimental); $\lambda_{0}$, central wavelength; $P$, initial peak power of pulse; $N_{\text {sol }}$, soliton order; $t_{1}$, initial FWHM pulse width; $t_{\max }$, maximum time-domain size; $M_{\text {sam }}$, number of grid points in time; $L_{\mathrm{LD}}$, dispersion length; $L_{\mathrm{N}}$, nonlinear length; $L_{\mathrm{S}}$, shock length; $z_{\max }$, maximum fiber length; $M_{\mathrm{stp}}$, maximum step number. Values replaced with "_- are tabulated or explained elsewhere. The conditions for the initial temporal electric-field profile such as the ratio of the initial backward wave to the forward wave $B / F$ (Sec. IV B), the initial coefficient related to the chirp $C_{0}$ (Sec. IV E), and the initial phase of the envelop wave relative to the carrier wave $\phi_{\mathrm{I}}(\mathrm{Sec}$. VI) are shown by 0.0 in the column "Ini.," only in the case that all of them are zero.

\begin{tabular}{|c|c|c|c|c|c|c|c|c|c|c|c|c|c|c|c|c|c|c|c|}
\hline Figs. & Cs. & Mtd. & Pro. & Rng. & Nres. & Ndis. & Ldis. & $\begin{array}{l}\text { Ini. : } \phi_{\mathrm{I}} \\
(\operatorname{rad} / \pi)\end{array}$ & $\begin{array}{c}\lambda_{0} \\
(\mathrm{~nm})\end{array}$ & $\begin{array}{c}P \\
(\mathrm{~kW})\end{array}$ & $N_{\text {sol }}$ & $\begin{array}{c}t_{1} \\
\text { (fs) }\end{array}$ & $\begin{array}{l}t_{\max } \\
(\mathrm{fs})\end{array}$ & $M_{\mathrm{sam}}$ & $\begin{array}{c}L_{\mathrm{LD}} \\
(\mathrm{mm})\end{array}$ & $\begin{array}{c}L_{\mathrm{N}} \\
(\mathrm{mm})\end{array}$ & $\begin{array}{c}L_{\mathrm{S}} \\
(\mathrm{mm})\end{array}$ & $\begin{array}{c}z_{\max } \\
(\mathrm{mm})\end{array}$ & $M_{\mathrm{stp}}$ \\
\hline 2 & - & FD & 2 & A & D & - & $\mathrm{S}_{\mathrm{m}}$ & $\mathrm{E}:-$ & 79 & 175 & 3.30 & 12.00 & 400 & 2048 & 1.427 & 0.131 & 0.868 & 2.500 & 30000 \\
\hline 3 & Sld & FD & 2 & A & D & SE & $\mathrm{S}_{\mathrm{m}}$ & $\mathrm{G}: 0.0$ & 800 & - & - & - & 500 & 2048 & - & - & - & 1.000 & 80000 \\
\hline 3 & Dsh & SG & 1 & B & I & SF & 2 & $\mathrm{G}: 0.0$ & 800 & - & - & - & 500 & 2048 & - & - & - & 1.000 & 80000 \\
\hline 3 & Dot & SG & 1 & B & I & $\mathrm{F}$ & 2 & $\mathrm{G}: 0.0$ & 800 & - & - & - & 500 & 2048 & - & - & - & 1.000 & 80000 \\
\hline 5 & - & - & - & - & - & - & - & S :0.0 & 830 & 2290 & 4.78 & 5.00 & 500 & 2048 & 0.238 & 0.010 & 0.029 & 1.000 & 80000 \\
\hline 7,8 & - & FD & 2 & A & D & SE & $\mathrm{S}_{\mathrm{m}}$ & $\mathrm{G}:-$ & 800 & 6870 & 5.87 & 3.40 & 500 & 2048 & 0.115 & 0.003 & 0.006 & 0.006 & 800 \\
\hline 10 & - & FD & 2 & A & I & SF & - & G :0.0 & 800 & - & - & - & 500 & 2048 & - & - & - & - & 20000 \\
\hline 11 & - & FD & 2 & A & - & - & $\mathrm{S}_{\mathrm{m}}$ & S :0.0 & 830 & 2290 & 4.78 & 5.00 & 500 & 2048 & 0.238 & 0.010 & 0.029 & 1.000 & 80000 \\
\hline 12 & - & FD & 2 & A & D & - & $\mathrm{S}_{\mathrm{m}}$ & $\mathrm{G}: 0.0$ & 800 & 2290 & 4.99 & 5.00 & 500 & 2048 & 0.249 & 0.010 & 0.028 & 1.000 & 80000 \\
\hline
\end{tabular}

tended NLSE (28) simultaneously with (12a) and confirming their reliability by comparing their solutions.

Since $\mathbf{F}_{\mathrm{L}}, \mathbf{F}_{\mathrm{N}}$, and $\mathbf{A}$ are quantities in the frequency domain, linear, and nonlinear frequency dispersions are naturally incorporated, and the causes of numerical troubles are easily traced. Since the temporal electric-field profile $E(t)$ and the intensity spectrum $I(\nu)$ are obtained at each spatial point, they are directly compared with experimental results.

In comparison with the FDTD method, the FDM is stable for long propagation distances under wide range of analytic parameter values. We confirmed it by solving the same onedimensionalized Maxwell's equations (2) under the same set of conditions and parameter values, and by comparing their relative errors and calculation times, as shown in Appendix B.

In the following, conditions and parameter values for each result are tabulated in Table I. Values replaced with "_-" are tabulated or explained elsewhere. Symbols $A, B, C$, etc., in each figure and table mean cases of different conditions whereas (a), (b), (c), (d), etc., just indicate subfigures or subtables.

The central wavelength is limited around $\lambda_{0}=800 \mathrm{~nm}$, the initial FWHM pulse width is $t_{1}=2.67-12.00 \mathrm{fs}$, and the initial peak power of pulse ranges widely as $P=25-6870 \mathrm{~kW}$, which is below the damage threshold $6 \mathrm{MW}$ for the input pulse width shorter than $6 \mathrm{fs}$ [22]. We chose these values considering our current and future experimental conditions. The temporal electric-field profile is initially Gaussian in most cases, and sech (hyperbolic secant) or experimental profiles are sometimes used for comparison.

\section{QUANTITATIVE INVESTIGATION ON CONVENTIONAL ASSUMPTIONS AND APPROXIMATIONS}

Before showing main results by the FDM concerning fewoptical-cycle pulse propagation in a fused-silica fiber, we summarize here some results obtained by preliminary calculations.

\section{A. Size effect of the periodic time domain}

Linear and nonlinear dispersions sometimes broaden temporal electric-field profiles over the maximum time-domain size $t_{\max }$ after long distance propagation. Since the Fourier transform employs the periodic boundary condition, these profiles overlap with themselves across the right and left boundary. For the intense broad case of $\left(P, t_{1}\right)=(5725$, $\mathrm{kW}, 10.00 \mathrm{fs})$ and the medium case of $(2290 \mathrm{~kW}, 5.00 \mathrm{fs})$ corresponding to $A$ and $E$ of Fig. 3, below, we compared the conditions used for $I_{1}(\nu)$ with $\left(t_{\max }, M_{\text {sam }}\right)=(1000 \mathrm{fs}, 4096)$ and $I_{2}(\nu)$ with $(500 \mathrm{fs}, 2048)$, where $M_{\text {sam }}$ is the number of grid points in time.

From the difference of $I_{1}(\nu)$ and $I_{2}(\nu)$, we found that $t_{\max }$ larger than $500 \mathrm{fs}$ (and $M_{\text {sam }}$ larger than 2048) is necessary for the intense broad case whereas we can use $\left(t_{\text {max }}, M_{\text {sam }}\right)$ $=(500 \mathrm{fs}, 2048)$ as the sufficient size for the medium case .

\section{B. Effect of the bidirectional propagation}

Since the present FDM is based on the bidirectional propagation, we can consider the interaction of forward and backward waves, and the validity of the unidirectional propa- 
gation approximation. From this standpoint, we compared temporal profiles and intensity spectra of the forward wave calculated with and without the unidirectional propagation approximation, which is realized under $F_{\mathrm{N}}$ of Eq. (22a) where $b_{1}(\xi, \tau)$ is approximated by $a_{1}(\xi, \tau)$. This means we neglect the action of the backward wave on the forward wave.

We compared the results for some values of the initial amplitude ratio of the backward wave to the forward wave $B / F$. In the case of $B / F=0.0$, we had expected that the backward wave would be generated naturally after many periodic overlaps with the forward wave in the periodic time domain. However, the amplitude of the backward wave was less than $10^{-4}$ of that of the forward wave after propagation. In the case of $B / F=1.0$, the backward wave propagates as if independent of the forward wave, and the difference between the intensity spectra with and without the unidirectional propagation was slight.

When the backward wave leaves fast enough from the forward wave as now, the interaction between forward and backward waves is slight, and using the unidirectional propagation approximation could be justified if desired. However, in other cases with closer group velocities between the forward wave and the backward wave, the FDM employing the bidirectional propagation is certainly important.

\section{Effect of the restricted frequency range}

While deriving the NLSE, we neglected the spectral components outside of the basic range $-2 \omega_{0}<\omega<2 \omega_{0}$. In the numerical approach, this situation is retrieved by filtering the spectral components of $F_{\mathrm{N}}$ in (12a) only in the basic range. The difference of the temporal electric-field profiles and the intensity spectra between without and with such a filter is slight as long as the initial width of the spectrum is narrow and the initial peak power is low enough to confine the intensity spectrum within the basic range after propagation. Under the condition that this effect can be found, however, the calculation itself breaks down. As the result, such a filter is inappropriate.

\section{Effect of the linear dispersion}

A conventional treatment of the linear dispersion has been approximating the propagation constant $\beta(\omega)$ by polynomials in $\omega-\omega_{0}$. However, in cases of broad frequency spectra of pulses as of now, it is required to use the correct linear dispersion as given by the modified Sellmeier equation (16). As for the intensity spectrum $I(\nu)$ and the spectral phase $\phi(\nu)$, we compared their aspects calculated without and with this approximation for the linear dispersion under the same soliton order 1.04 and $\xi=z_{\max } / L_{\mathrm{LD}}=35.2$, where $z_{\max }$ and $L_{\mathrm{LD}}$ are the maximum fiber length and the dispersion length (Appendix C).

The spectra $I(\nu)$ by the propagation constant which is quadratic in $\omega-\omega_{0}$ approximate rather better those in the Sellmeier case whereas $I(\nu)$ by the higher-order dispersion up to the fifth order are totally different. When the initial peak power is high, a satellite appears in the lower frequency

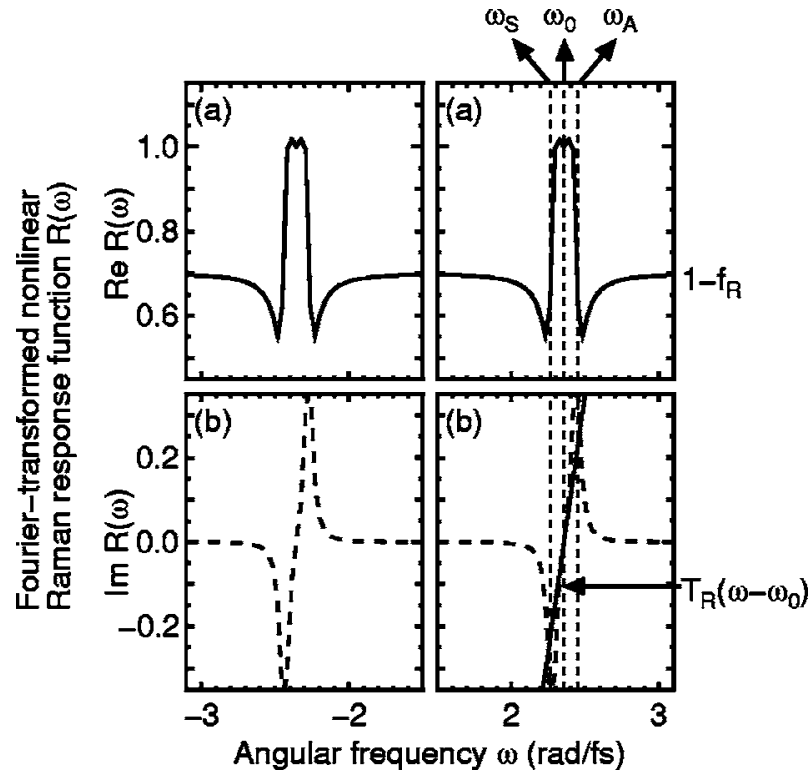

FIG. 1. Fourier-transformed nonlinear Raman response function $\tilde{R}(\omega)$, (a) real part and (b) imaginary part. The range $-1.5 \mathrm{rad} / \mathrm{fs}$ $<\omega<1.5 \mathrm{rad} / \mathrm{fs}$ is omitted. A linear approximation to its imaginary part around $\omega_{0}$ is also shown. $\omega_{\mathrm{S}}=2.274 \mathrm{rad} / \mathrm{fs}, \omega_{0}=2.356 \mathrm{rad} / \mathrm{fs}$, $\omega_{\mathrm{A}}=2.438 \mathrm{rad} / \mathrm{fs}$, and $T_{\mathrm{R}}=2.44 \mathrm{fs}$.

range of $I(\nu)$ in the Sellmeier case. This satellite is considered to be the resonance line where the spectral phase $\phi(\nu)$ reaches an extremum, and the group velocity vanishes. It is concluded that the propagation constant approximated by polynomials in $\omega-\omega_{0}$ cannot be used anymore, and the correct linear dispersion is indispensable. This is true especially when optical pulses are intense and short, and their spectra are broad, as now.

\section{E. Effects of the delayed Raman response and nonlinear dispersions}

Figure 1 shows the Fourier transform of the delayed Raman nonlinear response function $\widetilde{R}(\omega)$. It is unity at the central frequency $\omega_{0}$, but approaches $1-f_{\mathrm{R}}$ outside of the Stokes frequency $\omega_{\mathrm{S}}=2.274 \mathrm{rad} / \mathrm{fs}$ or the anti-Stokes frequency $\omega_{\mathrm{A}}=2.438 \mathrm{rad} / \mathrm{fs}$, reducing effective nonlinear effects. According to Eq. (29d), the gradient of its imaginary part at $\omega_{0}$ is $T_{\mathrm{R}}=2.44 \mathrm{fs}$, which gives rise to the infinite Raman selffrequency shift in the extended NLSE (28).

To determine the core dispersion, we consulted the experimental input spectrum $I_{\text {in }}(\nu)$ and the experimental output spectrum $I_{\text {out }}(\nu)$, as shown by $D$ and $C$ in Fig. 2 . These were generated by launching optical pulses with $\left(P, t_{1}\right)$ $=(175 \mathrm{~kW}, 12.00 \mathrm{fs})$ and $\lambda_{0}=798 \mathrm{~nm}$ into a polarizationpreserving single-mode fused-silica fiber (Newport, F-SP-V, the length $z=2.5 \mathrm{~mm}$, the core radius $a=1.35 \mu \mathrm{m}$, and the numerical aperture $\left.N_{\mathrm{A}}=0.16\right)[23,24]$. In the inset of Fig. 2, $A(B)$ shows the experimental (theoretical) scaled effective core radius $w(\omega) / a$ as a function of the normalized frequency $V(\omega) \equiv N_{\mathrm{A}} a \omega / c$. We modified $B$, the theoretical core dispersion [25], in various ways mainly in the range 1.5 


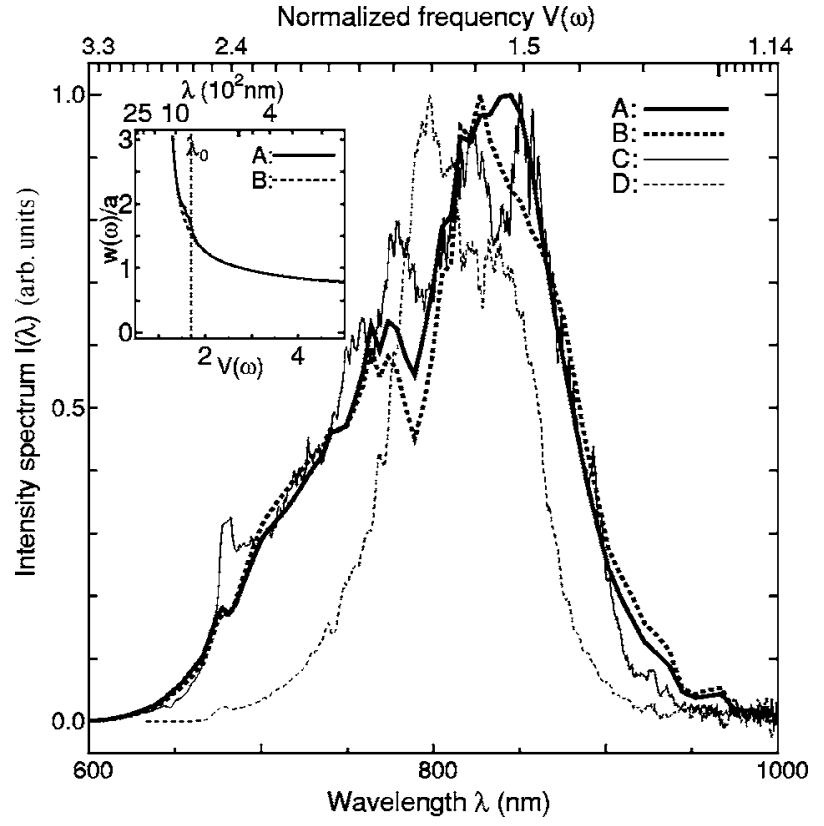

FIG. 2. Intensity spectra used for the investigation of the core dispersion. $A$ or $B$ : Calculated spectrum under the experimental or theoretical effective core radius as far as $z=2.5 \mathrm{~mm}$ where $B / F$ $=0.0, \phi_{1}=0.0$, and $C_{0}=-12.0 \mathrm{rad} \mathrm{fs}^{2} . C$ : Output experimental spectrum $I_{\text {out }}$ compared with $A$ or $B . D$ : Input experimental spectrum $I_{\text {in }}$ used as the initial condition. Inset: Variation of experimental or theoretical $(A, B)$ effective core radius $w(\omega) / a$ with the normalized frequency $V(\omega) \equiv N_{\mathrm{A}} a \omega / c$.

$<V(\omega)<2.0(900 \mathrm{~nm}>\lambda>680 \mathrm{~nm})$. With each of these core dispersions, we calculated the output spectrum $I(\nu)$ at $z=2.5 \mathrm{~mm}$, starting with the initial temporal electric-field profile $\widetilde{E}(z=0, \omega) \propto \sqrt{I_{\text {in }}(\nu)} \exp \left[i C_{0}\left(\nu-\nu_{0}\right)^{2}\right]$, where $C_{0}=$ $-12.0 \mathrm{rad} \mathrm{fs}^{2}$ is the coefficient related to the linear downchirp. Then, we chose the one which fits best to $I_{\text {out }}(\nu)$. As $A$ in Fig. 2 shows, $I(\nu)$ obtained in this way fits better than $B$ obtained by the theoretical core dispersion. The experimental core dispersion used then is $A$ in the inset. In the range shorter than the central wavelength of $I(\nu)$, subpeaks appear or disappear rather sensitively to the effective core radius $w(\omega)$. In this range, $w(\omega)$ was determined to be neither too large nor too small. When it is large and hence the nonlinearity is reduced, the spectrum is hard to change. When it is small, on the other hand, spectral components pass through this range too fast toward the higher frequency range and no subpeaks appear. Further difference between $I(\nu)$ and $I_{\text {out }}(\nu)$ may be attributed to the axial asymmetry of the panda fiber used for this experiment. In the low frequency range, however, the spectrum is rather insensitive to $w(\omega)$.

The combined effect of the delayed Raman response and the core dispersion is investigated in Appendix D, under the same set of conditions as Kalosha-Herrmann (hereafter K-H) [8], $\lambda_{0}=830 \mathrm{~nm},\left(P, t_{1}\right)=(2290 \mathrm{~kW}, 5.00 \mathrm{fs})$, and $z=1.0 \mathrm{~mm}$. The effect of the delayed Raman response is small irrespective of the core dispersion. Under the present condition of the broad initial spectrum and the short propagation distance, only the slight deformation of the spectrum arises instead of the self-frequency shift. In the nonlinear dispersion, the self-steepening forms the basic structure of the spectrum with a sharp peak and a broad wing. After the core dispersion is introduced, furthermore, the satellite in the lower frequency disappears, the main peak sharpens, the shoulder in the higher frequency appears, and the wing stretches. They are caused by the nonlinear effects decreased in the lower frequency range, and increased in the higher frequency range by the core dispersion. The spectral phase is rather insensitive to these effects.

\section{SUBFEMTOSECOND PULSE COMPRESSION}

\section{A. Analysis at different initial peak powers and pulse widths}

Figure 3 shows the intensity spectra $I(\nu)$, spectral phases $\phi(\nu)$, and the temporal electric-field profiles $E(t)$ at the propagation distances $z=0.1 / 0.5 \mathrm{~mm}$ which were calculated under the initial peak power $P=763 / 2290 / 5725 \mathrm{~kW}$, and the initial pulse width $t_{1}=10.00 / 5.00 / 2.67 \mathrm{fs}$. We compare here results by the FDM [solid curve, Eq. (12)] with those by the NLSE with the self-steepening [dashed curve, Eq. (28) without the term of $\left.T_{\mathrm{R}}\right]$ and those by the NLSE without the self-steepening [dotted curve, Eq. (31)]. In some cases of large $P$, the results by the NLSE with the self-steepening are not shown due to the breakdown of calculation. As for $E(t)$, only the results by the FDM are shown for simplicity. Part of $\phi(\nu)$ is sometimes omitted in the range where $I(\nu)$ is weak.

The nonlinear length $L_{\mathrm{N}}$, the shock length $L_{\mathrm{S}}$ $=0.39 L_{\mathrm{N}} \omega_{0} t_{1}$ [the case of the Gaussian envelope of the temporal electric-field profile $E(t)]$, and the dispersion length $L_{\mathrm{LD}}$ are measures for nonlinear effects (SPM and selfsteepening) and the linear dispersion. These characteristic lengths are directly calculated from the initial peak power $P$ and the initial FWHM pulse width $t_{1}$. Figure 4(a) tabulates $L_{\mathrm{LD}}, L_{\mathrm{N}}$, and $L_{\mathrm{S}}$ for the cases in Fig. 3. Figure 4(b) shows these lengths for $t_{1}=10.0 \mathrm{fs}\left(L_{\mathrm{LD}}=0.995 \mathrm{~mm}\right)$ and $P$ $=25 / 100 / 350 / 500 \mathrm{~kW}\left(L_{\mathrm{N}}=0.917 / 0.229 / 0.065 / 0.046 \mathrm{~mm}\right)$ in addition. Theoretically, results by the NLSE agree with those by the FDM at the propagation distance $z$ below the shortest among $L_{\mathrm{LD}}, L_{\mathrm{N}}$, and $L_{\mathrm{S}}$, which is actually the nonlinear length $L_{\mathrm{N}}$ in all the cases shown. At the propagation distance $z=0.1 \mathrm{~mm}$, it was confirmed that all of $I(\nu), \phi(\nu)$, and $E(t)$ obtained by the NLSE and those by the FDM agree well for $P=25 / 100 \mathrm{~kW}$ since $z<L_{\mathrm{N}}$, and differ slightly for $P=350 / 500 \mathrm{~kW}$ since $z \geqslant L_{\mathrm{N}}$.

Nonlinear effects appear in all the cases in Fig. 3, because $P>500 \mathrm{~kW}$ and even $z=0.1 \mathrm{~mm}$ satisfies $z>L_{\mathrm{N}}$ $=0.030 / 0.010 / 0.004 \mathrm{~mm}$ [see Fig. 4(a)].

The propagation distance $z=0.5 \mathrm{~mm}$ exceeds the shock length $L_{\mathrm{S}}=0.006-0.166 \mathrm{~mm}$ in all the cases, but $z=0.1 \mathrm{~mm}$ does not except for $G,\left(P, t_{1}\right)=(763 \mathrm{~kW}, 10.00 \mathrm{fs})$.

The linear dispersion appears or does not appear depending on $t_{1}$. The dispersion length is $L_{\mathrm{LD}}=0.995 / 0.249 /$ $0.071 \mathrm{~mm}$ for $t_{1}=10.00 / 5.00 / 2.67 \mathrm{fs}$ [see Fig. 4(a)]. Then, at $z=0.1 / 0.5 \mathrm{~mm}$, the linear dispersion appears if $t_{1}=2.67 \mathrm{fs}$ $\left(C, F, I\right.$ in Fig. 3) since $z>L_{\mathrm{LD}}=0.071 \mathrm{~mm}$, but it does not if $t_{1}=10.00 \mathrm{fs}\left(A, D, G\right.$ in Fig. 3) since $z<L_{\mathrm{LD}}=0.995 \mathrm{~mm}$.

Thus, the effects of the SPM, the self-steepening, and the linear dispersion are expected to appear with propagation in 
(a)
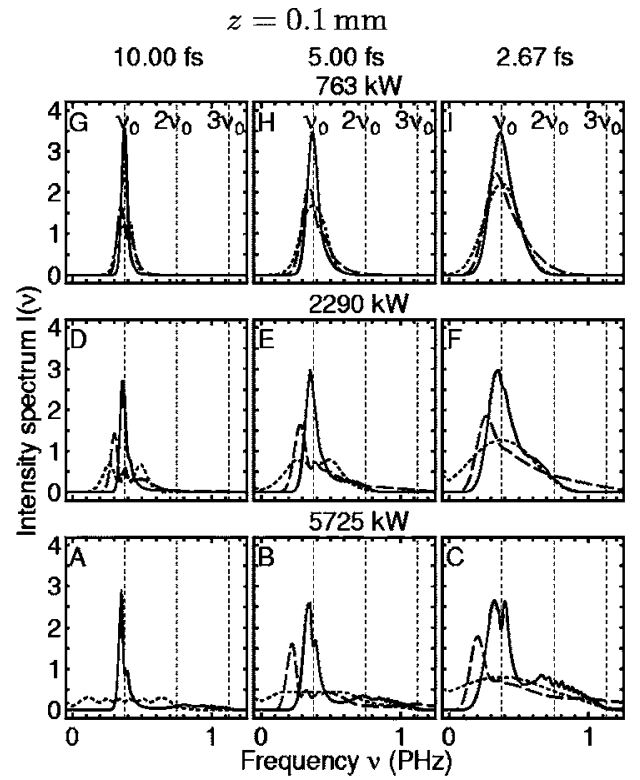

$763 \mathrm{~kW}$

(b)

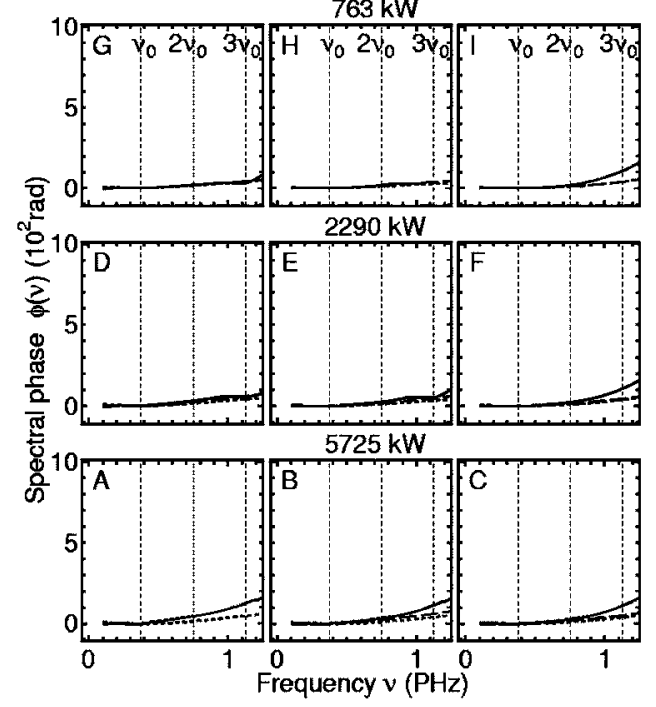

(c)

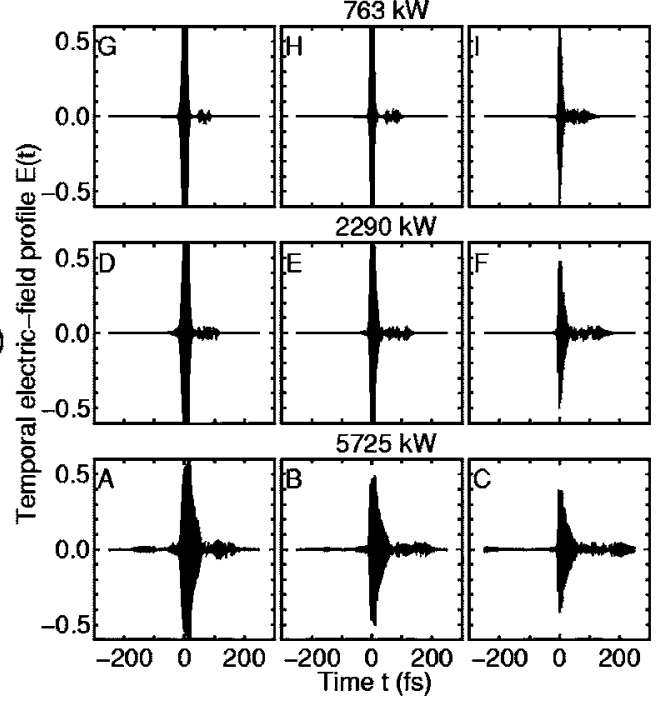

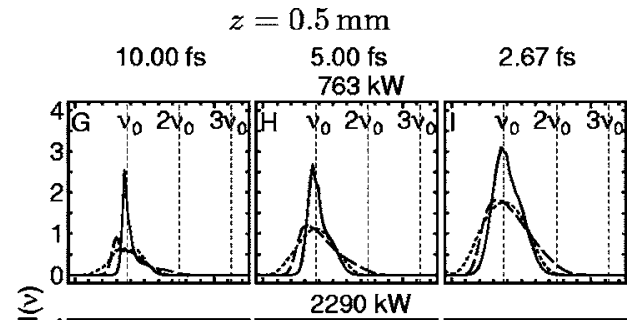
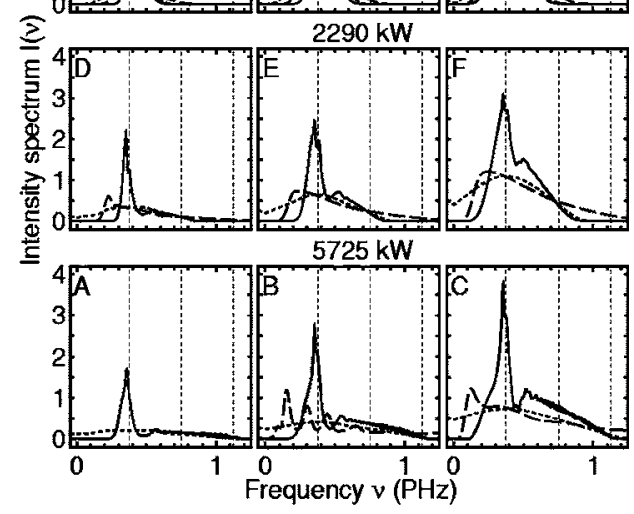

$763 \mathrm{~kW}$
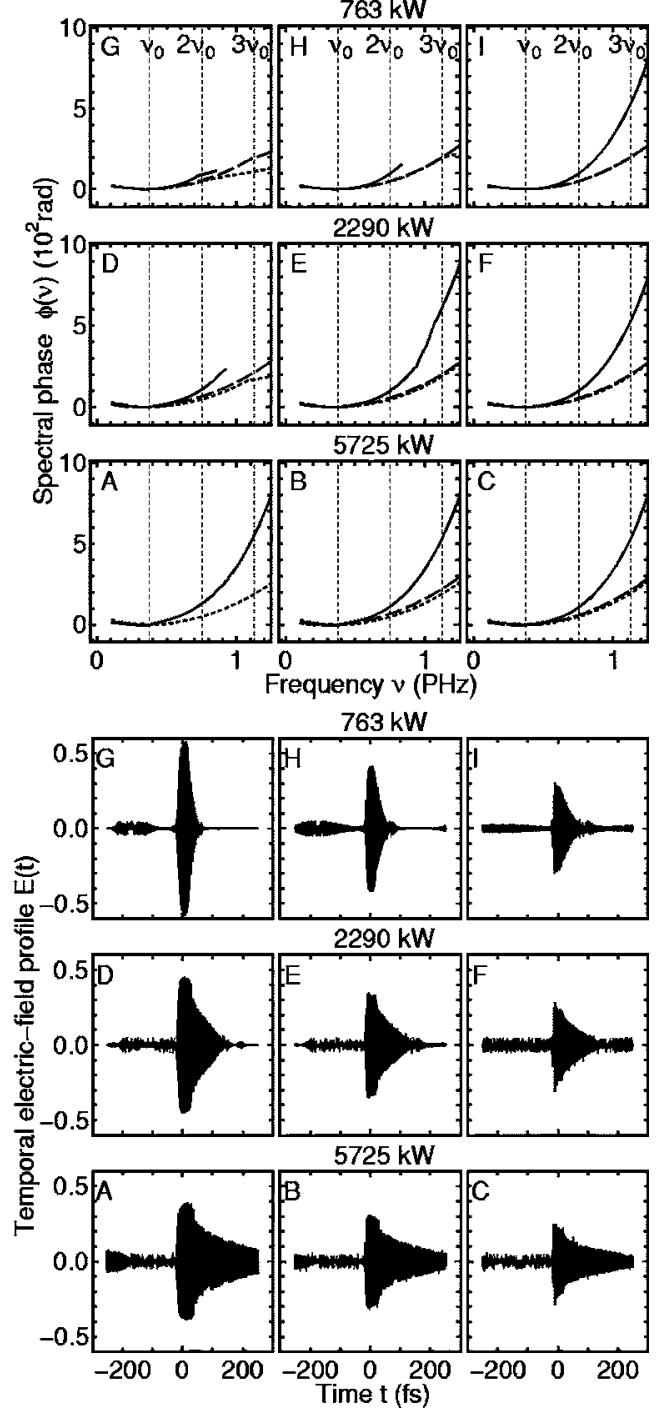

FIG. 3. Dependence of (a) intensity spectrum $I(\nu)$, (b) spectral phase $\phi(\nu)$, and (c) temporal electric-field profile $E(t)$ on the initial peak power and the pulse width at $z=0.1 \mathrm{~mm}$ (left) and $z=0.5 \mathrm{~mm}$ (right). Those calculated by the FDM, NLSE with the self-steepening, and NLSE without the self-steepening are shown by solid curves, dashed curves, and dotted curves, respectively. 


\begin{tabular}{ccccccccc}
\hline \hline Fig. Cs. & $\begin{array}{c}P \\
(\mathrm{~kW})\end{array}$ & $N_{\mathrm{sol}}$ & $\begin{array}{c}t_{1} \\
(\mathrm{fs})\end{array}$ & $\begin{array}{c}L_{\mathrm{LD}} \\
(\mathrm{mm})\end{array}$ & $\begin{array}{c}L_{\mathrm{N}} \\
(\mathrm{mm})\end{array}$ & $\begin{array}{c}L_{\mathrm{S}} \\
(\mathrm{mm})\end{array}$ \\
\hline 3 & A & 5725 & 15.76 & 10.00 & 0.995 & 0.004 & 0.022 \\
3 & $\mathrm{~B}$ & 5725 & 7.88 & 5.00 & 0.249 & 0.004 & 0.011 \\
3 & $\mathrm{C}$ & 5725 & 4.21 & 2.67 & 0.071 & 0.004 & 0.006 \\
3 & $\mathrm{D}$ & 2290 & 9.97 & 10.00 & 0.995 & 0.010 & 0.055 \\
3 & $\mathrm{E}$ & 2290 & 4.99 & 5.00 & 0.249 & 0.010 & 0.028 \\
3 & $\mathrm{~F}$ & 2290 & 2.66 & 2.67 & 0.071 & 0.010 & 0.015 \\
3 & $\mathrm{G}$ & 763 & 5.76 & 10.00 & 0.995 & 0.030 & 0.166 \\
3 & $\mathrm{H}$ & 763 & 2.88 & 5.00 & 0.249 & 0.030 & 0.083 \\
3 & I & 763 & 1.54 & 2.67 & 0.071 & 0.030 & 0.044 \\
\hline \hline
\end{tabular}

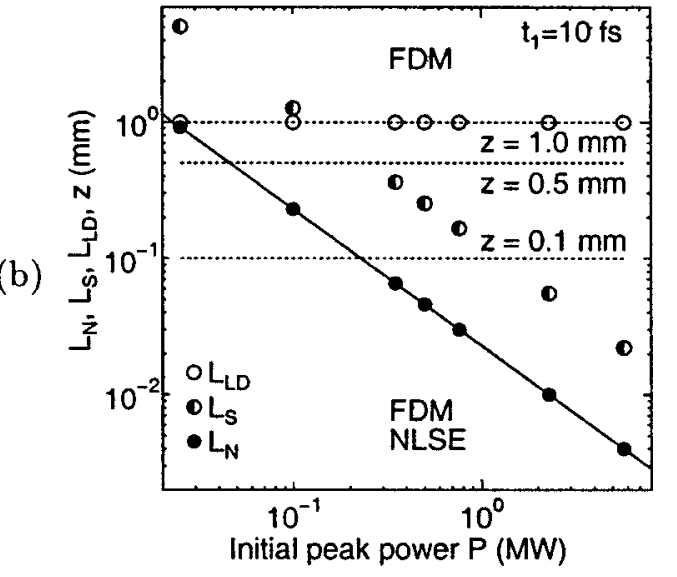

FIG. 4. Nonlinear length $L_{\mathrm{N}}$, shock length $\mathrm{L}_{\mathrm{S}}$, and dispersion length $L_{\mathrm{LD}}$ (full / half-full / empty circles) as functions of the initial peak power $P$ and the initial FWHM pulse width $t_{1}\left[t_{1}=10.0 \mathrm{fs}\right.$ for (b)]. Below $L_{\mathrm{N}}$, the NLSE is applicable as well as the FDM. Refer to Table I for headings and abbreviations in (a).

this order for the parameter values of $P$ and $t_{1}$ chosen here.

At $z=0.1 \mathrm{~mm}$, we observe differences in intensity spectra $I(\nu)$ obtained by the FDM and those by the NLSE in all the cases in Fig. 3, as expected from $z>L_{N}$. In contrast to $I(\nu)$ obtained by the NLSE without the self-steepening, those obtained by the FDM are asymmetric with respect to the central frequency $\nu_{0}$, and diminish in the lower frequency. They have a sharp main peak just below $\nu_{0}$ and a broad wing in the higher frequency range. For larger $P$, such a structure stands out more, but the shift of the main peak and the broadening of the wing are reduced by the delayed Raman response and the core dispersion, compared with $I(\nu)$ obtained by the NLSE with the self-steepening. The main peak splits in the frequency range where the core dispersion changes significantly with the frequency (below $\lambda_{0}$ in the inset of Fig. 2).

Among the spectral phases $\phi(\nu)$ obtained by the FDM, and the NLSE with and without the self-steepening, those by the FDM increase most significantly with increasing $\left|\nu-\nu_{0}\right|$.

In temporal electric-field profiles $E(t)$, we find a main pulse with the front part steeper than the rear part, and following wave packets with small amplitude. The structure of
$I(\nu)$ with a sharp peak around $\nu_{0}$ and a broad wing in the higher frequency range explains these aspects of $E(t)$ together with the central wavelength in the normal dispersion region. Actually, nearly coherent long waves come around the steeper front part, and random short waves compose the rear part and the wave packets following the main pulse.

Between $z=0.1 \mathrm{~mm}$ and $z=0.5 \mathrm{~mm}$, the change in $I(\nu)$ is larger for higher $P$. Though the main peak just broadens for lower $P(G, H$, and $I$ in Fig. 3), a hollow and a subpeak develop, for higher $P$, between the main peak and the wing due to the core dispersion ( $B, C, E$, and $F$ in Fig. 3). In addition, the split main peak merges and is sharpened, and the wing grows by the self-steepening $(B, C, E$, and $F$ in Fig. 3 ). These changes are slower for the FDM than the NLSE because of the nonlinearity reduced by the delayed Raman response and the core dispersion.

Spectral phases $\phi(\nu)$ at $z=0.5 \mathrm{~mm}$ have the similar aspects as those at $z=0.1 \mathrm{~mm}$. As for the change in $\phi(\nu)$ with $\left|\nu-\nu_{0}\right|$, it increases with propagation. Those by the FDM increase most significantly with increasing $\left|\nu-\nu_{0}\right|$. In addition, their quadratic profile in $\left|\nu-\nu_{0}\right|$ is mainly attributed to the linear dispersion which is shown in Fig. 10(b) of Appendix C.

Even before $z$ reaches $L_{\mathrm{LD}}$, the main pulse in $E(t)$ is already broad enough. As the result, the following small wave packets are involved in the main pulse. The intensity spectrum broadened by the nonlinearity induces the temporal dispersion combined with the fiber dispersion.

In $I(\nu)$ for a high $P$ at $z=1.0 \mathrm{~mm}$, we found a third-order harmonics around the frequency range higher than $2.6 \nu_{0}$. This third-order harmonics is attributed to the interaction of the main peak and the subpeak. However, in the present case if the delayed Raman response and the core dispersion reduce the nonlinearity, then its amplitude is much smaller than that predicted by Kalosha-Herrmann [8]. At $z=1.0 \mathrm{~mm}$, the changes in the spectral phases increase with propagation furthermore.

\section{B. Pulse compression by the complete phase compensation}

Optical pulses are compressed below the initial width by the complete phase compensation in addition to the spectral broadening by the SPM. When the electric-field profile just after propagated in a fiber is expressed as

$$
\tilde{E}\left(z_{\max }, \omega\right)=\left|\tilde{E}\left(z_{\max }, \omega\right)\right| \exp \left[i \phi\left(z_{\max }, \omega\right)\right],
$$

the field after the compressor is

$$
\tilde{E}_{c}(\omega)=\left|\tilde{E}\left(z_{\max }, \omega\right)\right| \exp \left[i \phi\left(z_{\max }, \omega\right)+i \phi_{c}(\omega)\right],
$$

with the phase compensated as $\phi\left(z_{\max }, \omega\right)+\phi_{c}(\omega)=0$. The temporal profile of the compressed pulse $E_{c}(t)$ is the inverse Fourier transform of $\widetilde{E}_{c}(\omega)$, and the temporal intensity $I_{c}(t)$ is proportional to $\left|E_{c}(t)\right|^{2}$.

In Fig. 5(a), we compare $E_{c}(t)$ obtained by the FDM with those by Kalosha-Herrmann $(\mathrm{K}-\mathrm{H})[8]$ and by the NLSE at $z_{\max }=0.1 / 0.5 / 1.0 \mathrm{~mm}$, under the same condition of $\left(P, t_{1}\right)$ $=(2290 \mathrm{~kW}, 5.00 \mathrm{fs})$. Figure 5(c) shows the dependence of the FWHM $t_{c}$ measured from $I_{c}(t)$ on the fiber length. 
(a)

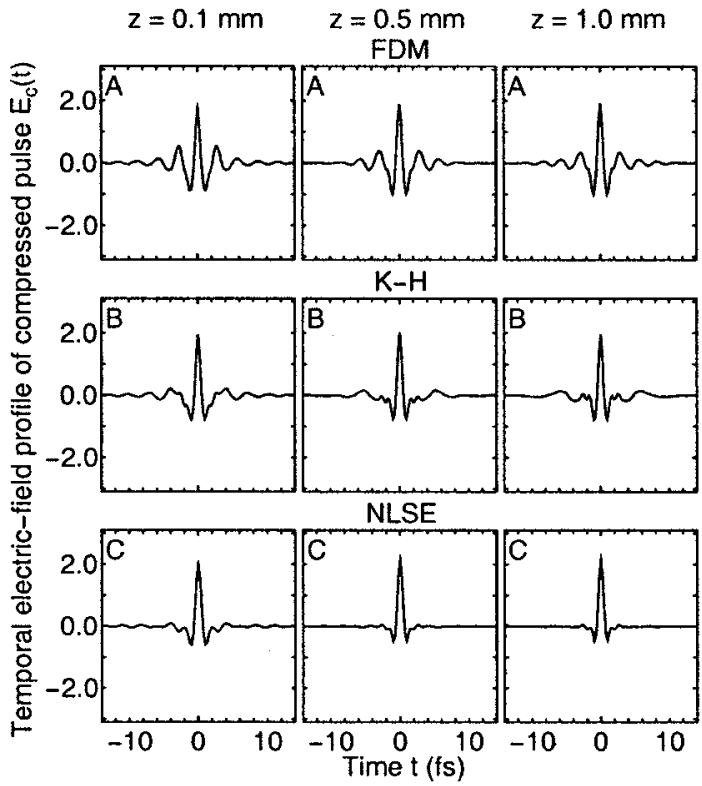

(b)

(c)

\begin{tabular}{cccccccc}
\hline \multicolumn{1}{c}{ Fig. Cs. Mtd. Pro. Rgn. Nres. Ndis. Ldis. } \\
\hline 5 & A & FD & 2 & A & D & SE & $\mathrm{S}_{\mathrm{m}}$ \\
5 & B & FD & 2 & A & I & SF & $\mathrm{S}_{\mathrm{m}}$ \\
5 & C & SG & 1 & B & I & F & 2 \\
\hline \hline
\end{tabular}

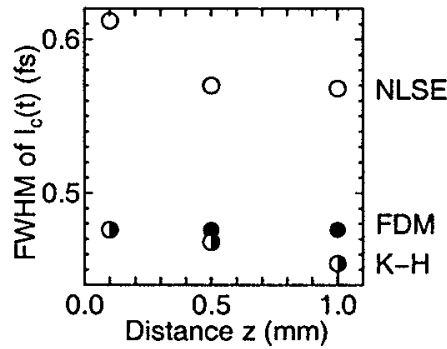

FIG. 5. (a) Temporal electric-field profile of the compressed pulse $E_{c}(t)$ for $\left(P, t_{1}\right)=(2290 \mathrm{~kW}, 5.00 \mathrm{fs})$ and $z_{\max }=0.1,0.5$, and $1.0 \mathrm{~mm}$ (left, center, right) calculated by FDM, K-H, and NLSE ( $A$, $B, C)$. (b) Refer to Table I for headings and abbreviations. (c) Dependence of $t_{c}$, the FWHM of the temporal intensity $I_{c}(t) \propto\left|E_{c}(t)\right|^{2}$, on the propagation distance. $E_{c}(t)$ in (a) are used, and $t_{c}$ by FDM, $\mathrm{K}-\mathrm{H}$, and NLSE are shown by full circles, half-full circles, and empty circles, respectively.

In addition to $t_{c}$ obtained from $I_{c}(t)$, we define here $t_{c}^{(\mathrm{e})}$ as the FWHM of the intensity obtained from the envelope of $E_{c}(t)$. In contrast to $t_{c}^{(\mathrm{e})}$ which is short when $I(\nu)$ broadens, $t_{c}$ is short when the mean frequency of $I(\nu)$ shifts toward the higher frequency.

As far as $t_{c}^{(\mathrm{e})}$ is concerned, the pulse calculated by the NLSE is shorter than that by the FDM, because $I(\nu)$ obtained by the NLSE broadens wider than that by the FDM, as shown in Fig. 3. However, $t_{c}$ calculated by the NLSE is longer, and $t_{c}$ by K-H is shorter than $t_{c}=0.476$ fs by the FDM which is almost independent of $z_{\max }$. In the NLSE without the self-steepening, $I(\nu)$ broadens symmetrically toward the lower frequency across $\nu=0$ as well as toward the higher frequency. Then, the central frequency of $I(\nu)$ lowers, and $t_{c}$ (a)

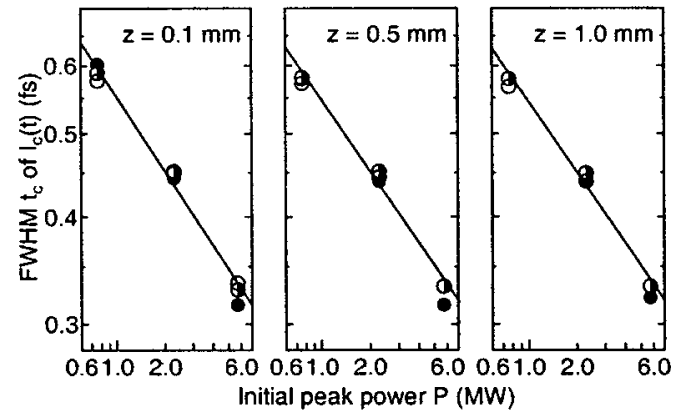

(b)

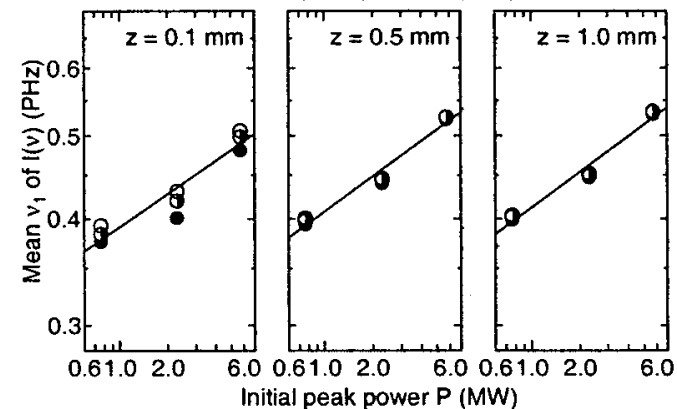

(c)
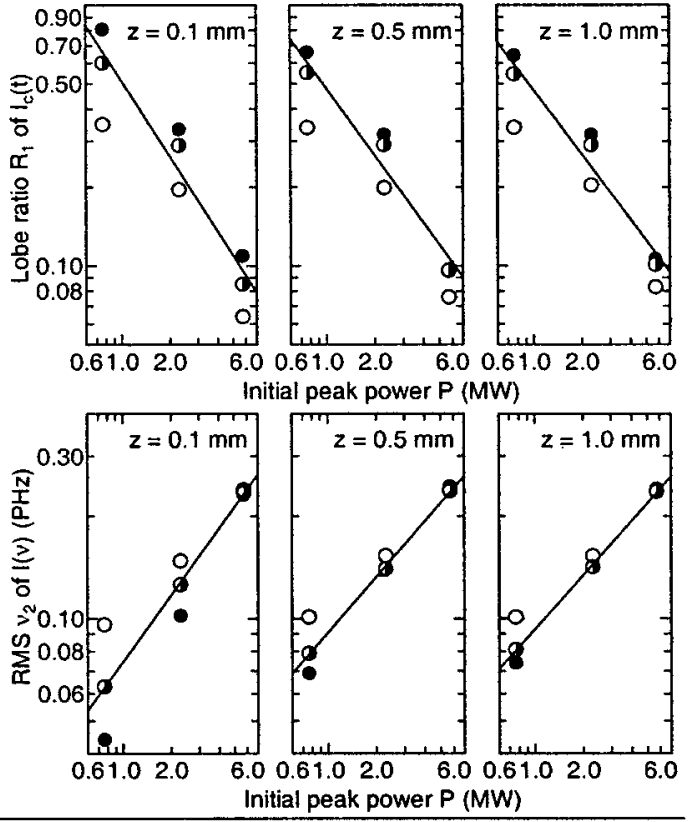

$\begin{array}{llllllllll}z(\mathrm{~mm}) & \alpha_{\mathrm{w}} & \beta_{\mathrm{w}} & \alpha_{1} & \beta_{1} & \alpha_{\mathrm{r}} & \beta_{\mathrm{r}} & \alpha_{2} & \beta_{2}\end{array}$

(e)

$0.100-0.594-0.287-0.9380 .128-0.667-0.960-2.600 .650$

$0.500-0.601-0.276-0.8970 .137-0.733-0.858-2.400 .544$

$1.000-0.605-0.274-0.8870 .139-0.743-0.828-2.380 .530$

FIG. 6. Dependence of the parameters in the temporal intensity of the compressed pulse $I_{c}(t)$ and the intensity spectrum $I(\nu)$ of Fig. 3 on the initial peak power $P(\mathrm{MW})$ and the initial pulse width $t_{1}$. (a) FWHM $t_{c}$ of $I_{c}(t)(\mathrm{fs})$. (b) Mean $\nu_{1}$ of $I(\nu)(\mathrm{PHz})$. (c) Height of first lobe $R_{1}$ relative to main peak in $I_{c}(t)$. (d) RMS $\nu_{2}$ of $I(\nu)(\mathrm{PHz})$. Those for the initial pulse width $t_{1}=10.00,5.00$, and $2.67 \mathrm{fs}$ are shown by full circles, half-full circles, and empty circles, respectively. They are fitted against $P$ as $t_{c}=e^{\alpha_{\mathrm{w}}} P^{\beta_{\mathrm{w}}}, \nu_{1}=e^{\alpha_{1}} P^{\beta_{1}}, R_{1}$ $=e^{\alpha_{\mathrm{r}}} P^{\beta_{\mathrm{r}}}$, and $\nu_{2}=e^{\alpha_{2}} P^{\beta_{2}}$ for $t_{1}=5.00 \mathrm{fs}$ and $z_{\max }=0.1 / 0.5 / 1.0 \mathrm{~mm}$. Fit parameters are tabulated in (e). 
is longer than those obtained by the other numerical methods. Contrarily, in K-H's results where neither the delayed Raman response nor the core dispersion reduce the nonlinearity, the central frequency of $I(\nu)$ is kept higher, and $t_{c}$ is shorter than that by the FDM.

We obtained temporal intensities of the compressed pulses $I_{c}(t) \propto\left|E_{c}(t)\right|^{2}$ also from $I(\nu)$ in Fig. 3, and investigated their dependence on $P, t_{1}$, and $z_{\max }$. Figure 6(a) shows $t_{c}$ measured from $I_{c}(t)$ for $P=763 / 2290 / 5725 \mathrm{~kW}, t_{1}=10.00 / 5.00 /$ $2.67 \mathrm{fs}$, and $z_{\max }=0.1 / 0.5 / 1.0 \mathrm{~mm}$. The dependence of $t_{c}$ on $P$ is remarkable compared with $t_{1}$ and $z_{\max }$. Its decrease with $P$ is fitted well as $t_{c}=e^{\alpha_{\mathrm{w}}} P^{\beta_{\mathrm{w}}}$ with $\beta_{\mathrm{w}} \simeq-0.28$. The mean frequency

$$
\nu_{1} \equiv \int_{0}^{\infty} d \nu I(\nu) \nu / \int_{0}^{\infty} d \nu I(\nu)
$$

of $I(\nu)$ in Fig. 6(b), changing as $\nu_{1}=e^{\alpha_{1}} P^{\beta_{1}}$ with $\beta_{1} \simeq 0.13$, is related with $t_{c}$. The height of the first lobe $R_{1}$ relative to the main peak in $I_{c}(t)$ is shown in Fig. 6(c). It also depends on $P$ most remarkably, on $t_{1}$ but more than $t_{c}$ in Fig. 6(a), and hardly on $z_{\max }$. The root-mean-square

$$
\nu_{2} \equiv \sqrt{\int_{0}^{\infty} d \nu I(\nu)\left(\nu-\nu_{1}\right)^{2} / \int_{0}^{\infty} d \nu I(\nu)}
$$

describing the width of $I(\nu)$ is shown in Fig. 6(d).

Shortness of the FWHM $t_{1}$ diminishes $R_{1}$, and refines the pulse quality of the compressed pulse profile [Fig. 6(c)]. But it hardly contributes to the compression of the pulse itself [Fig. 6(a)]. Both $t_{c}$ and $R_{1}$ are almost independent of $z_{\max }$ after $0.1 \mathrm{~mm}$ much longer than $L_{\mathrm{N}}$. As we observed in Fig. 3, $\phi(\nu)$ to be compensated increases with $z_{\max }$. Then, short $z_{\max }$ is preferable if we consider the limited range of the phase to be compensated by the compressor. Large $P$ refines the shortness and the pulse quality of the compressed pulse most effectively. The pulse is compressed to as short as $0.3 \mathrm{fs}$ at $P=6 \mathrm{MW}$.

\section{EFFECT OF THE CARRIER ENVELOPE PHASE}

It is expected that the carrier envelope phase (CEP), the initial phase of the carrier wave relative to the envelope wave of the electric field [26], influences the propagation behavior of few-optical-cycle pulses. We compare here propagation of pulses with different initial conditions as

$$
E^{j}(z=0, t)=\operatorname{Re} E_{+}(z=0, t) e^{-i\left(\omega_{0} t+\phi_{1}^{j}\right)}
$$

composed of only the forward wave $a_{1}(\xi, \tau)$ in Eq. (24), where we discriminate the different cases of CEP $\phi_{\mathrm{I}}^{j}=0, \pi / 2$, and $\pi$ by $j=A, B$, and $C$ here. Instead of the direct difference after propagation of a distance $z$, we use the function

$$
D^{j}(z, \omega) \equiv e^{i \phi_{\mathrm{F}}^{j} \widetilde{E}^{j}}(z, \omega)-e^{i \phi_{\mathrm{F}}^{A} \widetilde{E}^{A}(z, \omega)}
$$

which clearly extracts the essential difference caused by different initial CEPs. In this equation, $\widetilde{E}^{j}(z, \omega)=\mathrm{F} E^{j}(z, t)$ from Eq. (4) and the value of the restoration phase $\phi_{\mathrm{F}}^{j}$ is $\phi_{\mathrm{I}}^{j}(\omega$ $>0)$ or $-\phi_{\mathrm{I}}^{j}(\omega<0)$. We observe the difference between two

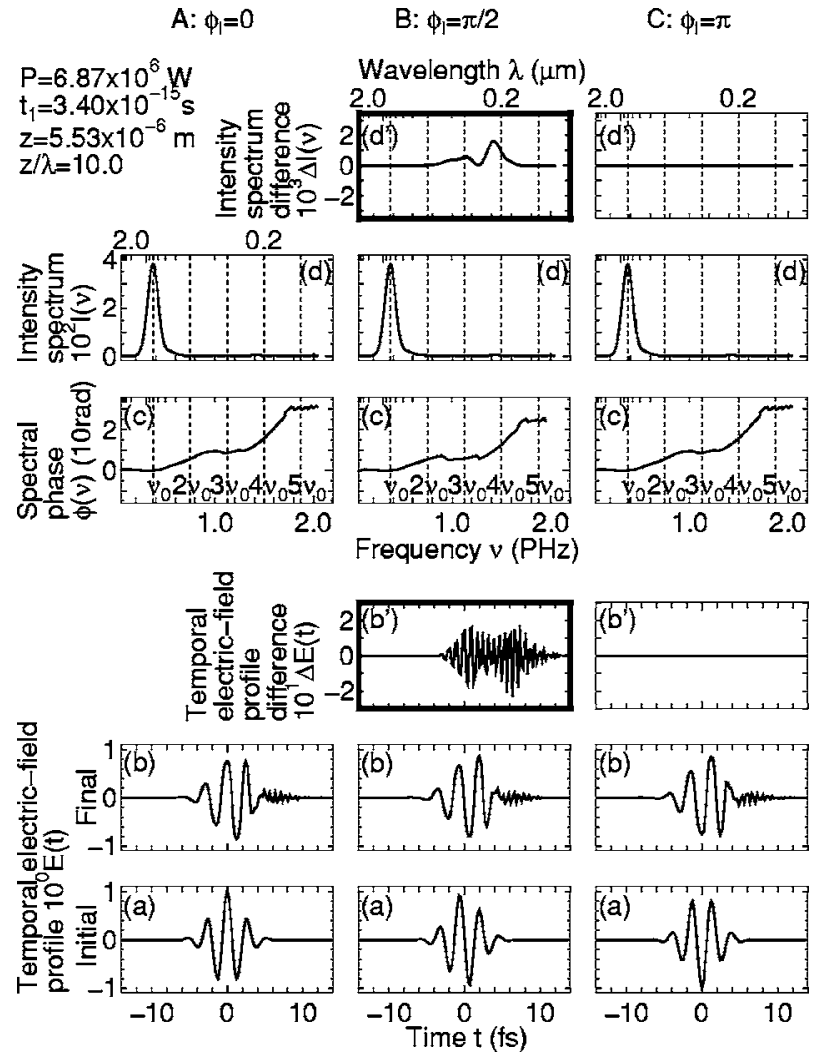

FIG. 7. Effect of the carrier envelope phase (CEP) for $\phi_{\mathrm{I}}=0$, $\pi / 2$, and $\pi(A, B$, and $C)$. (a) Initial temporal electric-field profile. (b) Temporal electric-field profile after propagation. ( $\left.\mathrm{b}^{\prime}\right)$ Temporal electric-field profile difference $\Delta E(t)$. (c) Spectral phase. (d) Intensity spectrum. $\left(\mathrm{d}^{\prime}\right)$ Intensity spectrum difference $\Delta I(\nu)$. In all cases, $\left(P, t_{1}\right)=(6870 \mathrm{~kW}, 3.40 \mathrm{fs}), \quad z=10 \lambda, B / F=0.0$, and $C_{0}$ $=0.0 \mathrm{rad} \mathrm{fs}^{2}$, respectively.

cases $j=A$ and $j \neq A$ by the temporal electric-field profile difference $\Delta E(t)=\mathrm{F}^{-1} D^{j}(z, \omega)$ and the spectral intensity difference $\Delta I(\nu)=\left|D^{j}(z, \omega)\right|^{2}$.

In (b), (c), and (d) of Fig. 7, we compare $E(t), \phi(\nu)$, and $I(\nu)$ of pulses with different $\phi_{\mathrm{I}}^{j}$ respectively, where the initial $E(t)$ is shown in (a). In $\left(\mathrm{b}^{\prime}\right)$ and $\left(\mathrm{d}^{\prime}\right), \Delta E(t)$ and $\Delta I(\nu)$ are shown, respectively. The condition of $\left(P, t_{1}\right)=(6870 \mathrm{~kW}$, $3.40 \mathrm{fs})$ and the propagation distance $z=10.0 \lambda$ are same where $\lambda=\lambda_{0} / n_{\mathrm{L}}^{\mathrm{R}}\left(\omega_{0}\right)$ is the wavelength in the fiber. Though the generation of the third-order harmonics is observed by a small wave packet composed of short waves in the rear part of $E(t)$ [Fig. 7(b)], the difference by the CEP is hard to observe. By contrast, $\Delta E(t)$ and $\Delta I(\nu)$ clearly show the differences between $A-B$ and $A-C$. We find no differences between $A$ and $C$ because they are just the sign inversion of the other concerning the electric field in the envelope wave. Then, we concentrate on the differences between $A$ and $B$.

Figure 8 shows the evolution of $\Delta E(t)$ and $\Delta I(\nu)$ between $\phi_{\mathrm{I}}^{A}=0$ and $\phi_{\mathrm{I}}^{B}=\pi / 2$ at propagation distances between $z / \lambda$ $=0.0$ and 10.0. This result was confirmed to be independent of the analytic conditions such as the change from $\left(t_{\max }, M_{\mathrm{sam}}\right)=(500 \mathrm{fs}, 2048)$ to $\left(t_{\max }, M_{\mathrm{sam}}\right)=(1000 \mathrm{fs}$, 4096). Before $z / \lambda=3.0$, a single wave packet in $\Delta E(t)$ and a peak around $3.0 \nu_{0}$ in $\Delta I(\nu)$ grow gradually with the propa- 


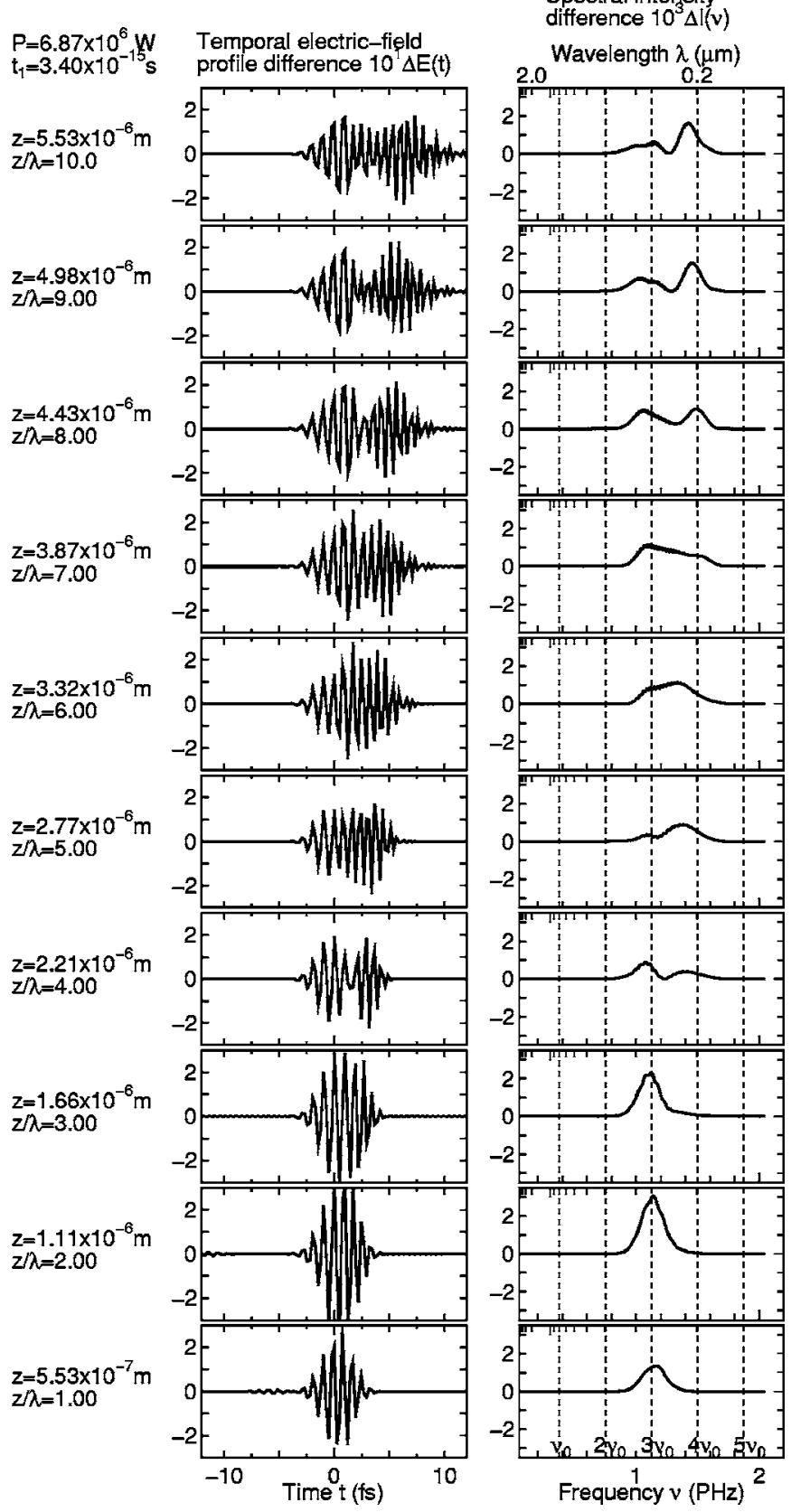

FIG. 8. Difference caused by the CEP between $\phi_{\mathrm{I}}=0$ and $\phi_{\mathrm{I}}$ $=\pi / 2$ for the respective propagation distances $z$. Left: temporal electric-field profile difference $\Delta E(t)$. Right: spectral intensity difference $\Delta I(\nu)$.

gation. We can attribute them to the third-order harmonics, because they do not appear when $\phi_{\mathrm{F}}^{j}$ is modified as $3 \phi_{\mathrm{I}}^{j}\left(2 \omega_{0}<\omega<4 \omega_{0}\right)$ or $-3 \phi_{\mathrm{I}}^{j}\left(-4 \omega_{0}<\omega<-2 \omega_{0}\right)$ in addition to $\phi_{\mathrm{I}}^{j}(\omega>0)$ or $-\phi_{\mathrm{I}}^{j}(\omega<0)$ to remove the difference in the range of the third-order harmonics. After $z / \lambda=3.0, \Delta E(t)$ tends to split into two wave packets, the faster of which has the longer oscillation period than the slower. In $\Delta I(\nu)$, components higher than the third-order harmonics appear, and two peaks around $3.0 \nu_{0}$ and $3.8 \nu_{0}$ change their height alternately distance by distance. Above $z / \lambda=10.0, \Delta E(t)$ disperses. In $\Delta I(\nu)$, the peak around $3.0 \nu_{0}$ splits into many low peaks while the one at $3.8 \nu_{0}$ remains unspread as far as $z / \lambda=1000.0$. These results clearly show that the CEP can be used as a tool to monitor the evolution of the nonlinear process.

Though the present numerical analysis takes into account the broad spectrum as well as the delayed Raman response and the core dispersion, we try to consider newly observed results of Fig. 8 here simply on the basis of the coherence length $l_{c}=\pi /\left[\beta\left(3 \omega_{0}\right)-3 \beta\left(\omega_{0}\right)\right]$, derived for the third-order nonlinear interaction of coherent waves. By use of the modified Sellmeier equation, $l_{c} / \lambda=n\left(\omega_{0}\right) / 6\left[n\left(3 \omega_{0}\right)-n\left(\omega_{0}\right)\right]$ is evaluated to be 4.3711 . Even if the wave generated by the nonlinear process under consideration is coherent at the propagation distance below $l_{c}$, it diminishes above $l_{c}$ by the phase mixing. Figure 8 shows the generation of the thirdorder harmonics from the initial wave around $\nu_{0}$ below $z / \lambda$ $=3.0$. Above this distance, the wave around $3.8 \nu_{0}$, which is considered to be generated from the initial wave and the third-order harmonics, begins to grow. Thus, the coherence length can be a measure of the distance for the generation of the higher-order harmonics also in the present analysis, though its estimation is rough due to the presence of the spectral components distributed continuously around $\nu_{0}$ in the initial $I(\nu)$.

To verify the above-found CEP effect, the following experiment is proposed. The beam from a few-optical-cycle source which we developed $[1,2]$ is split into two, and the CEP and the temporal delay is given to one of them. They are launched into an extremely short fiber with the time difference to avoid the interaction between them in the fiber. After the propagation in the fiber, the CEP and the temporal delay of these beams are restored, and their interference is observed. The broad spectral range up to the higher-order harmonics requires some attention to avoid the dispersion of the air and the resonance of the fiber material, and to calibrate the frequency-dependent characteristics of optical detectors. The fiber that is as short as some times of the wavelength of the pulse is also necessary. This new simple technique to measure and control the CEP will be valuable for upgrading the few-optical-cycle electric-field wavepacket source used for experiments.

\section{CONCLUSION}

For the nonlinear propagation analysis of monocycle optical pulses, the FDM was extended from the split-step Fourier method. The FDM is based on bidirectional propagation equations for forward and backward waves, which were derived directly from Maxwell's equations. The delayed Raman response and nonlinear dispersions (self-steepening, core dispersion) are naturally incorporated as well as the arbitrary linear dispersion. From one of the bidirectional propagation equations, the NLSE is shown to be derived straightforwardly, in contrast to the conventional derivation from the second-differential wave propagation equation by excessive approximations such as the SVEA. Furthermore, the initial condition of the unidirectional propagation is reasonably given.

We integrated the bidirectional propagation equations as multidimensional simultaneous equations evolved in space. 
We employed the exponential integral for the linear force whereas a more general method for the nonlinear force. The temporal electric-field profile and the intensity spectrum obtained at each spatial point are directly compared with experimental results. The present FDM was confirmed to be stable and accurate for long propagation distance under the wide range of analytic parameter values, in comparison with the FDTD method.

Influences and limitations of assumptions or approximations used to derive the NLSE were investigated: the restricted size of the time domain and the frequency domain, the unidirectional propagation approximation, the quadratic linear dispersion, the instantaneous response, and the neglected nonlinear dispersion. In the present analysis, not only the intensity spectrum but also the temporal electric-field profile sometimes broaden wide. The size of the time domain with the periodic boundary condition must cover wholly the broad pulse to avoid its overlap with itself across the boundaries. The size of the frequency domain, inversely proportional to the time-domain size, must be much broader than the basic frequency range $-2 \nu_{0}<\nu<2 \nu_{0}$ to avoid the numerical breakdown. To satisfy these contradictory requirements, the time-domain size $t_{\max }=500 \mathrm{fs}$ and the number of grid points in time $M_{\text {sam }}=2048$ were chosen, which cover the frequency domain broader than $\pm 5 \nu_{0}$. The unidirectional propagation approximation can be used instead of the bidirectional propagation, when the backward wave leaves from the forward wave fast enough.

The linear dispersion sensitively affects the widespread intensity spectrum of few-optical-cycle pulses as well as nonlinear effects (self-steepening, core dispersion, and delayed Raman response). A correct refractive index as given by the modified Sellmeier equation is important, since this index predicts a satellite caused by the vanishing group velocity in the low frequency range in contrast to the quadratic dispersion. The self-steepening forms the basic structure of the spectrum with a sharp peak around $\nu_{0}$ and a broad wing in the higher frequency range. The core dispersion increases the nonlinear effects in the higher frequency range, and decreases them in the lower frequency range. The delayed Raman response reduces the nonlinear effects and just modifies the spectrum.

Intensity spectra, spectral phases, and temporal electricfield profiles obtained by the FDM and those by the usual or extended NLSE were compared for the initial peak power $763 \mathrm{~kW}, 2290 \mathrm{~kW}, 5725 \mathrm{~kW}\left(L_{\mathrm{N}}=0.030 \mathrm{~mm}, 0.010 \mathrm{~mm}\right.$, $0.004 \mathrm{~mm}$ ) and the initial pulse width $10.00 \mathrm{fs}, 5.00 \mathrm{fs}$, and $2.67 \mathrm{fs}\left(L_{\mathrm{LD}}=0.995 \mathrm{~mm}, 0.249 \mathrm{~mm}, 0.071 \mathrm{~mm}\right)$ up to the propagation distance $z=1.0 \mathrm{~mm}$. For the parameter values as chosen above, the self-steepening, core dispersion, and the delayed Raman response appear as dominant, middle, and slight effects, respectively, on the intensity spectrum. Basic structure formed by the self-steepening stands out for the initial peak power larger than $2290 \mathrm{~kW}$. The core dispersion generates a subpeak in the frequency range higher than the main peak. In the FDM, the delayed Raman response and the core dispersion reduce the nonlinearity compared with the NLSE. As a result, the change of the intensity spectrum with the propagation such as the broadening of the main peak slows down.
In the temporal electric-field profile, we found a main pulse with the front part steeper than the rear part, and wave packets with a small amplitude following the main pulse. Nearly coherent long waves come around the steep front part of the main pulse, and random short waves compose its rear part and the wave packets because of the spectral structure and the central wavelength in the normal dispersion region. The main pulse broadens wide due to the dispersion induced by the nonlinear broadening of the spectrum even before $z$ reaches the linear dispersion length, and involves the small wave packets.

After the intensity spectrum is sufficiently broadened, optical pulses are compressed by the complete phase compensation. By choosing the initial peak power suitably, the FWHM $t_{c}$ of the temporal intensity of the compressed pulse is remarkably shortened, and its pulse quality is improved. By contrast, they are hardly changed by the initial pulse width, or by the propagation distance that is longer than 0.1 $\mathrm{mm}$. Among numerical methods, $t_{c}$ obtained by the FDM is shorter than that by the NLSE but a little longer than that by Kalosha-Herrmann [8].

It was demonstrated that the CEP does generate the difference in the temporal electric-field profile and the intensity spectrum, at the initial peak power of the order of megawatts. With increasing propagation distance, the difference appears in the third-order harmonics first, and grows in the higher-order harmonics range later, in parallel with the generation of higher-order harmonics induced by the nonlinear polarization. Experimental observation of the CEP will be achieved by mixing two beams of different CEPs and temporal delays with interference.

In Sec. II E, we clarified how the present FDM is reduced to the NLSE under some assumptions and approximations except for the SVEA. Furthermore, we confirmed the difference of the results obtained by both methods in Sec. V A, for several initial peak powers, initial pulse widths, and propagation distances. Such comparisons were quite efficient for developing the present FDM.

The FDM predicts that the femtosecond optical pulses propagated in conventional silica fibers will be compressed as short as $0.3 \mathrm{fs}$ at the damage threshold $P=6 \mathrm{MW}$. For the compression, larger initial peak power is more effective than shorter initial pulse width or longer fiber length. Then, the initial pulse need not be extremely short, and the fiber length is preferably short if we consider the spectral phase increasing over the limited dynamic range of the phase compensator.

The techniques to measure and control the CEP will serve for monitoring the evolution of nonlinear process as well as upgrading the few-optical-cycle electric-field wave packet source used for experiments. The importance of this FDM lies in the ability to investigate the significances, effects, and the limitations of the methods used for theories. Furthermore, it can predict phenomena or propose conditions for experiments on femtosecond optical pulses under the current or future extreme conditions, such as the coherent ultrawideband light generation in low power.

\section{ACKNOWLEDGMENTS}

The authors acknowledge Dr. R. Morita and Dr. N. Karasawa for valuable discussions. 


\section{APPENDIX A: DIRECT DERIVATION OF THE NONLINEAR SCHRÖDINGER EQUATION FROM BIDIRECTIONAL PROPAGATION EQUATIONS}

As seen from the second line of (12a), the backward wave $A_{2}$ can be generated from $A_{1}$ through $F_{\mathrm{N}}$ even if $A_{2}$ is absent initially. However, we can derive the NLSE only from the first line of (12a) for the forward wave with $\mathrm{EF}^{-1}$ operated on both sides:

$$
\begin{gathered}
\mathrm{EF}^{-1} \frac{\partial A_{1}}{\partial \xi}=\mathrm{EF}^{-1}\left(F_{\mathrm{L} 1}-F_{\mathrm{N}}\right), \\
F_{\mathrm{L} 1} \equiv Z\left(i \beta-\frac{i \omega}{v}\right) A_{1}, \\
F_{\mathrm{N}}=-i \gamma_{\mathrm{FDM}}(\omega) \mathrm{F} b_{1}(\xi, \tau) \mathrm{F}^{-1} \widetilde{R}(\omega) \mathrm{F}\left[b_{1}(\xi, \tau)\right]^{2}, \\
\gamma_{\mathrm{FDM}}(\omega)=\frac{\omega Z}{c} \frac{\chi^{(3)}}{2 n_{\mathrm{L}}^{\mathrm{R}}(\omega)} \frac{P}{c \varepsilon_{0} n_{\mathrm{L}}^{\mathrm{R}}(\omega) A_{\mathrm{eff}}(\omega)} .
\end{gathered}
$$

From Eq. (24a), the left side is

$$
\mathrm{EF}^{-1} \frac{\partial A_{1}}{\partial \xi}=\mathrm{F}^{-1} Z\left(\frac{\partial A_{+}}{\partial z}+i \beta_{0} A_{+}\right)=\frac{\partial a_{+}}{\partial \xi}+i \beta_{0 \mathrm{C}} a_{+} .
$$

The propagation constant $\beta(\omega)$ in Eq. (A1b) is expanded into the Taylor series around $\omega_{0}$ as

$$
\beta(\omega)=\sum_{n=0}^{\infty} \frac{\beta_{n}}{n !}\left(\omega-\omega_{0}\right)^{n}, \quad \beta_{n} \equiv \beta^{(n)}\left(\omega_{0}\right) .
$$

Such an expansion is valid as long as the spectrum of the pulse is localized around $\omega_{0}$. When $A_{1}$ is Fourier-transformed inversely together with $-i \omega$,

$$
\mathrm{EF}^{-1}(-i \omega) A_{1}=\frac{1}{T} \mathrm{E} \frac{\partial a_{1}}{\partial \tau}=\frac{1}{T}\left(\frac{\partial a_{+}}{\partial \tau}-i \omega_{0 \mathrm{C}} a_{+}\right)
$$

from Eq. (24a). After transposing the second term on the right side and extending the equation to the higher-order differentiation, we rewrite this relation as

$$
\mathrm{EF}^{-1}\left(\omega-\omega_{0}\right)^{n} A_{1}=\left(\frac{i}{T} \frac{\partial}{\partial \tau}\right)^{n} a_{+} .
$$

Then, the linear term (A1b) becomes

$$
\begin{aligned}
\mathrm{EF}^{-1} F_{\mathrm{L} 1}= & \mathrm{F}^{-1} Z(i \beta-i \omega / v) A_{+} \\
= & Z \mathrm{EF}^{-1}\left[i \sum_{n=0}^{\infty} \frac{\beta_{n}}{n !}\left(\omega-\omega_{0}\right)^{n}-\frac{i \omega}{v}\right] A_{1} \\
= & Z\left[i \sum_{n=0}^{\infty} \frac{\beta_{n}}{n !}\left(\frac{i}{T} \frac{\partial}{\partial \tau}\right)^{n} a_{+}+\frac{1}{v T}\left(\frac{\partial a_{+}}{\partial \tau}-i \omega_{0 \mathrm{C}} a_{+}\right)\right] \\
\simeq & -\left(\beta_{1 \mathrm{C}}-\frac{Z / T}{v}\right) \frac{\partial a_{+}}{\partial \tau}-\frac{i \beta_{2 \mathrm{C}}}{2} \frac{\partial^{2} a_{+}}{\partial \tau^{2}} \\
& -\frac{i(Z / T) \omega_{0 \mathrm{C}}}{v} a_{+}+i \beta_{0 \mathrm{C}} a_{+},
\end{aligned}
$$

where higher-order terms than the second order in $\omega-\omega_{0}$ are neglected in the last equation, and $\beta_{0 \mathrm{C}} \equiv Z \beta_{0}, \beta_{1 \mathrm{C}} \equiv Z \beta_{1} / T$, $\beta_{2 \mathrm{C}} \equiv Z \beta_{2} / T^{2}=Z / L_{\mathrm{LD}}$, and the dispersion length $L_{\mathrm{LD}} \equiv T^{2} / \beta_{2}$ are defined.

When the nonlinear response is almost instantaneous, $\widetilde{R}(\omega)$ is approximated, by the use of Eq. (A5), as

$$
\begin{gathered}
\mathrm{F}^{-1} \tilde{R}(\omega) \mathrm{F} \simeq \mathrm{F}^{-1}\left[1+\widetilde{R}^{(1)}\left(\omega-\omega_{0}\right)\right] \mathrm{F}=1-\frac{T_{\mathrm{R}}}{T} \frac{\partial}{\partial \tau} \\
T_{\mathrm{R}} \equiv \frac{1}{i} \widetilde{R}^{(1)}=\int_{0}^{\infty} r(t) t d t=\frac{2 f_{\mathrm{R}} \tau_{1}^{2} \tau_{2}}{\tau_{1}^{2}+\tau_{2}^{2}}
\end{gathered}
$$

where only terms up to the first order in $\omega-\omega_{0}$ are left in Eq. (A7a), and $\widetilde{R}^{(1)} \equiv[\mathrm{d} \widetilde{R}(\omega) / \mathrm{d} \omega]_{\omega=\omega_{0}}$.

Furthermore, we assume that the nonlinear dispersion is weak. Then, we expand $\gamma_{\mathrm{SPM}}(\omega) \equiv(3 / 4) \gamma_{\mathrm{FDM}}(\omega)$ into the Taylor series in $\omega-\omega_{0}$, and leave only terms up to the first order as

$$
\begin{gathered}
\mathrm{F}^{-1} \gamma_{\mathrm{SPM}}(\omega) \mathrm{F} \simeq \mathrm{F}^{-1}\left[\gamma_{\mathrm{SPM}}^{(0)}+\gamma_{\mathrm{SPM}}^{(1)}\left(\omega-\omega_{0}\right)\right] \mathrm{F} \\
=\gamma_{\mathrm{SPM}}^{(0)}+\frac{i \gamma_{\mathrm{SPM}}^{(1)} \frac{\partial}{\partial \tau}}{T} \\
\gamma_{\mathrm{SPM}}^{(0)} \equiv \gamma_{\mathrm{SPM}}\left(\omega_{0}\right)=Z \frac{\omega_{0}}{c} \frac{3 \chi^{(3)}}{8 n_{0}}\left[\widetilde{E}_{\mathrm{p}}\left(\omega_{0}\right)\right]^{2}=\frac{Z}{L_{\mathrm{N}}}, \\
L_{\mathrm{N}} \equiv\left[\frac{\omega_{0}}{c} \frac{3 \chi^{(3)}}{8 n_{0}} \frac{P}{c \varepsilon_{0} n_{0} A_{\mathrm{eff}}\left(\omega_{0}\right)}\right]^{-1}, \\
\gamma_{\mathrm{SPM}}^{(1)} \equiv\left[\frac{\mathrm{d} \gamma_{\mathrm{SPM}}(\omega)}{\mathrm{d} \omega}\right]_{\omega=\omega_{0}},
\end{gathered}
$$

where $L_{\mathrm{N}}$ is the nonlinear length. In the case of the unidirectional propagation approximation, $B_{1} \simeq A_{1}$ and $b_{1} \simeq a_{1}$. In addition, we drop terms proportional to $e^{ \pm 3 i \omega_{0 \mathrm{C}} \tau}$ while using Eq. (24a), which restricts the frequency for $F_{\mathrm{N}}$ in the basic range $-2 \omega_{0}<\omega<2 \omega_{0}$. After all these approximations, the nonlinear term (A1c) is rewritten as

$$
\mathrm{EF}^{-1}\left(-F_{\mathrm{N}}\right)=i \mathrm{EF}^{-1} \gamma_{\mathrm{FDM}}(\omega) \mathrm{F} b_{1}(\xi, \tau) \mathrm{F}^{-1} \widetilde{R}(\omega) \mathrm{F}\left[b_{1}(\xi, \tau)\right]^{2}
$$

$$
\begin{aligned}
\simeq & i \mathrm{EF}^{-1} \gamma_{\mathrm{FDM}}(\omega) \mathrm{F} \\
& \times \frac{3}{8}\left[a_{+}(\xi, \tau) e^{i\left(\beta_{0 \mathrm{C}} \xi-\omega_{0 \mathrm{C}} \tau\right)} \mathrm{F}^{-1} \widetilde{R}(\omega) \mathrm{F}\left|a_{+}(\xi, \tau)\right|^{2}\right. \\
& \left.+a_{+}^{*}(\xi, \tau) e^{i\left(-\beta_{0 \mathrm{C}} \xi+\omega_{0 \mathrm{C}} \tau\right)} \mathrm{F}^{-1} \tilde{R}(\omega) \mathrm{F}\left|a_{+}(\xi, \tau)\right|^{2}\right] \\
& \quad(\mathrm{A} 9 \mathrm{~b}) \\
\simeq & i \mathrm{~F}^{-1} \gamma_{\mathrm{SPM}}(\omega) \mathrm{F} a_{+}(\xi, \tau) \mathrm{F}^{-1} \tilde{R}(\omega) \mathrm{F}\left|a_{+}(\xi, \tau)\right|^{2}
\end{aligned}
$$




$$
\begin{aligned}
& \simeq i\left(\gamma_{\mathrm{SPM}}^{(0)}+\frac{i \gamma_{\mathrm{SPM}}^{(1)}}{T} \frac{\partial}{\partial \tau}\right) \\
& \quad \times a_{+}(\xi, \tau)\left(1-\frac{T_{\mathrm{R}}}{T} \frac{\partial}{\partial \tau}\right)\left|a_{+}(\xi, \tau)\right|^{2} .
\end{aligned}
$$

We use Eqs. (A2), (A6), and (A9d) in Eq. (A1a). Then, we derive the extended NLSE (28) and the usual NLSE (31).

Conventional derivation of the nonlinear Schrödinger equation usually begins with the wave propagation equation

$$
\frac{\partial^{2} \widetilde{E}}{\partial z^{2}}+\beta^{2} \widetilde{E}=-\frac{\omega^{2}}{c^{2}} \mathrm{~F} \frac{P_{\mathrm{N}}}{\varepsilon_{0}}=-\frac{2 i \beta E_{\mathrm{p}}}{Z} F_{\mathrm{N}}
$$

with the nonlinear term expressed by $(12 b)$, which is derived by eliminating $\tilde{H}(z, \omega)$ between the equations in (6). The Fourier transform of the electric field $\widetilde{E}(z, \omega)$ in the left side of Eq. (A10) is expressed as

$$
\begin{aligned}
\tilde{E}(z, \omega)= & E_{\mathrm{p}}(\omega) B_{1}\left(\xi, \omega_{\mathrm{C}}\right)=E_{\mathrm{p}}(\omega)\left[A_{1}\left(\xi, \omega_{\mathrm{C}}\right)+A_{2}\left(\xi, \omega_{\mathrm{C}}\right)\right] \\
= & \frac{E_{\mathrm{p}}(\omega)}{2}\left\{\left[A_{+}\left(\xi, \omega_{\mathrm{C}}-\omega_{0 \mathrm{C}}\right)+A_{-}\left(\xi, \omega_{\mathrm{C}}+\omega_{0 \mathrm{C}}\right)\right] e^{i \beta_{0 \mathrm{C}} \xi}\right. \\
& \left.+\left[A_{+}^{*}\left(\xi,-\omega_{\mathrm{C}}-\omega_{0 \mathrm{C}}\right)+A_{-}^{*}\left(\xi,-\omega_{\mathrm{C}}+\omega_{0 \mathrm{C}}\right)\right] e^{-i \beta_{0 C} \xi}\right\} \\
\equiv & \frac{E_{\mathrm{p}}(\omega)}{2}\left[B_{+}(z, \omega) e^{i \beta_{0} z}+B_{-}(z, \omega) e^{-i \beta_{0} z}\right]
\end{aligned}
$$

by (9d), (11b), and (25). Accordingly, $B_{+}$is the Fouriertransformed envelope of the scaled electric field, and $E B_{1}$ $=B_{+}$and $\mathrm{E}\left(\partial B_{1} / \partial z\right)=\partial B_{+} / \partial z+i \beta_{0} B_{+}$hold.

We operate $E F^{-1} Z /\left(2 i \beta E_{\mathrm{p}}\right)$ on all sides of $(\mathrm{A} 10)$. The third side of Eq. (A10) becomes $\mathrm{EF}^{-1}\left(-F_{\mathrm{N}}\right)$. This is no other than (A9) leading to the nonlinear terms in the extended NLSE (28). On the other hand, the first side of Eq. (A10) becomes rigorously as follows:

$$
\begin{aligned}
\mathrm{EF}^{-1} & \frac{Z}{2 i \beta E_{\mathrm{p}}}\left(\frac{\partial^{2} \widetilde{E}}{\partial z^{2}}+\beta^{2} \widetilde{E}\right) \\
& =\mathrm{EF}^{-1} \frac{Z}{2 i \beta}\left(\frac{\partial^{2} B_{1}}{\partial z^{2}}+\beta^{2} B_{1}\right) \\
& =\mathrm{EF}^{-1} \frac{Z}{2 i \beta}\left[2 i \beta\left(\frac{\partial}{\partial z}-i \beta\right) B_{1}+\left(\frac{\partial}{\partial z}-i \beta\right)^{2} B_{1}\right] \\
& =\mathrm{F}^{-1} Z\left\{\left[\frac{\partial}{\partial z}-i\left(\beta-\beta_{0}\right)\right] B_{+}+\frac{1}{2 i \beta} \mathcal{R}\right\},
\end{aligned}
$$

where the residual term $\mathcal{R}$ is defined as follows:

$$
\mathcal{R} \equiv \mathrm{E}\left(\frac{\partial}{\partial z}-i \beta\right)^{2} B_{1}=\left[e^{i\left(\beta-\beta_{0}\right) z} \frac{\partial}{\partial z} e^{-i\left(\beta-\beta_{0}\right) z}\right]^{2} B_{+} .
$$

Under the assumption of infinitesimal $\beta-\beta_{0}$, the residual term $\mathcal{R}$ approximately equals to $\partial^{2} B_{+} / \partial z^{2}$. In comparison with the first term in (A12), $\mathcal{R}$ is neglected in agreement with the SVEA. Then, Eq. (A12) agrees with the difference between (A2) and (A6) which leads to the linear terms in the extended NLSE (28), if $B_{+}$is set equal to $A_{+}$by the unidirectional propagation approximation $A_{-} \simeq 0$.

\section{APPENDIX B: ACCURACY AND EFFICIENCY OF THE FDM COMPARED WITH THE FDTD METHOD}

We compare here the accuracy and the efficiency of the Fourier direct method and the FDTD method, by solving the one-dimensionalized Maxwell's equations (2) under the same set of conditions and parameter values.

The essential difference of these methods lies in the treatment of linear terms either by the Fourier transform or by the finite difference. Furthermore, the FDTD method treats the linear dispersion and the nonlinear term in a way rather specialized to the model [13]. Thus we neglect these effects here. We put $\beta(\omega)=\beta_{1} \omega$ or $n_{\mathrm{L}}=c \beta_{1}$, omit $P_{\mathrm{N}}$ in Eq. (6), and we move on to the moving coordinate system by $(9 \mathrm{~b})$. Then, we obtain

$$
\begin{gathered}
\frac{\partial E}{\partial z}=\frac{1}{v} \frac{\partial E}{\partial t}+\frac{1}{c} \frac{\partial H}{\partial t}, \\
\frac{\partial H}{\partial z}=\frac{1}{v} \frac{\partial H}{\partial t}+\frac{n_{\mathrm{L}}^{2}}{c} \frac{\partial E}{\partial t},
\end{gathered}
$$

where $-c \mu_{0} H$ is replaced with $H$.

Equation (B1) is no other than the inverse Fourier transform of Eqs. (10a) and (10b) when $n_{\mathrm{L}}$ is replaced with $c \beta_{1}$, and $G_{\mathrm{N}}$ is neglected. Instead of Eq. (12a) in the FDM, (B1) is rigorously solved for $E$ as

$$
E_{\text {rig }}(z, t)=g_{+}\left[t+\left(\frac{1}{v}-\frac{n_{\mathrm{L}}}{c}\right) z\right]+g_{-}\left[t+\left(\frac{1}{v}+\frac{n_{\mathrm{L}}}{c}\right) z\right],
$$

where $g_{+}(t)$ and $g_{-}(t)$ are initial profiles of the forward wave and the backward wave, respectively. On the other hand, (B1) is discretized as

$$
\begin{gathered}
H_{i+1 / 2}^{n+1 / 2}=H_{i+1 / 2}^{n-1 / 2}+\frac{\Delta z}{v \Delta t}\left(H_{i+1}^{n-1 / 2}-H_{i}^{n-1 / 2}\right)+\frac{n_{\mathrm{L}}^{2} \Delta z}{c \Delta t}\left(E_{i+1}^{n}-E_{i}^{n}\right), \\
E_{i}^{n+1}=E_{i}^{n}+\frac{\Delta z}{v \Delta t}\left(E_{i+1 / 2}^{n}-E_{i-1 / 2}^{n}\right)+\frac{\Delta z}{c \Delta t}\left(H_{i+1 / 2}^{n+1 / 2}-H_{i-1 / 2}^{n+1 / 2}\right)
\end{gathered}
$$

to obtain $E_{i}^{n+1}$ at the temporal point $i \Delta t$ and the spatial point $(n+1) \Delta z$ by the FDTD method, where interpolated quantities are used to calculate the terms proportional to $\Delta z / v \Delta t$.

Under the same set of parameter values used for $B$ in Fig. 3 , we calculated $E(z, t)$ of (B1) by the FDM and the FDTD method as far as $z_{\max } / 100$ measuring the calculation time. Then, we compared both results with $E_{\text {rig }}(z, t)$ in (B2). Figure 9(a) shows the relative error between $E(z, t)$ and $E_{\text {rig }}(z, t)$ defined by 
(a)

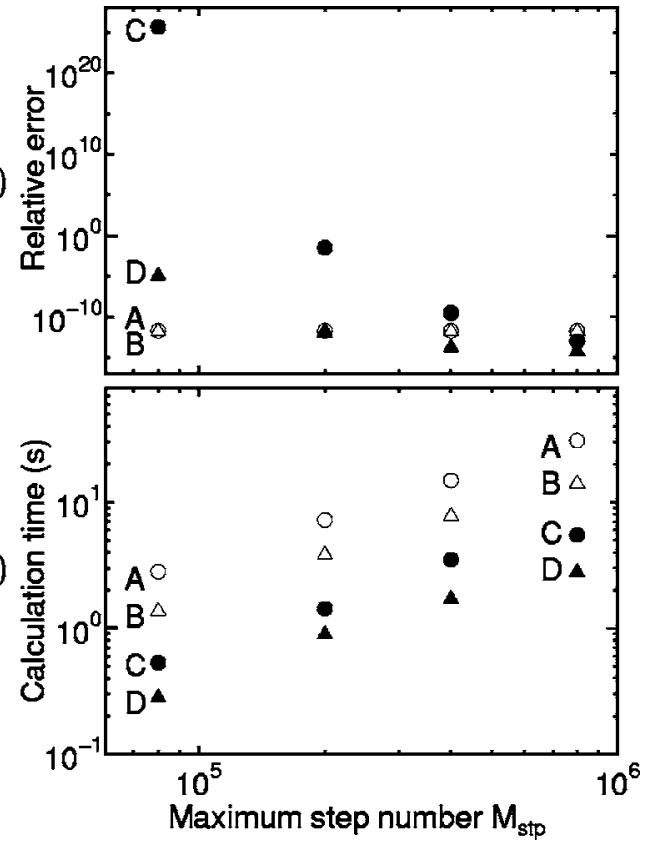

FIG. 9. Dependence of (a) relative error and (b) calculation time on the maximum step number $M_{\text {stp }}$ to obtain $E(z, t)$ at $z_{\max } / 100$ from a set of one-dimensionalized Maxwell's equations with neither the linear dispersion nor the nonlinear term: $A$, by the FDM with $M_{\text {sam }}=2048 ; B$, by the FDM with $M_{\text {sam }}=1024 ; C$, by the FDTD method with $M_{\text {sam }}=2048$; and $D$, by the FDTD method with $M_{\text {sam }}=1024$. In all cases, $t_{\max }=500 \mathrm{fs}$ and $z_{\max }=1.0 \mathrm{~mm}$.

$$
\delta \equiv \int_{-\infty}^{\infty} d t\left[E(z, t)-E_{\mathrm{rig}}(z, t)\right]^{2} / \int_{-\infty}^{\infty} d t\left[E_{\mathrm{rig}}(z, t)\right]^{2}
$$

At $\left(M_{\text {sam }}, M_{\text {stp }}\right)=(2048,80000)$ used in Fig. 3 , the FDM (A) already achieves the relative error as low as the rounding-off error. This error is almost independent of $M_{\text {stp }}$ when it is larger than 80000 . By contrast, the relative error of the FDTD method $(C)$ is extremely large there because of the instability growing rapidly with the propagation distance. It decreases with $M_{\text {stp }}$, however, and reaches the level of the FDM around $M_{\text {stp }}=400000$.

At the same $M_{\text {stp }}$, the FDM spends about five times as much calculation time as the FDTD method [Fig. 9(b)], which is mainly attributed to the exponential integral for attaining the stability. However, at the same error level, the calculation time of both methods almost balance after all.

In contrast to the FDM $[A, B$ of Fig. 9(a)], $\delta$ of the FDTD method $(C, D)$ depends on $M_{\text {sam }}$ considerably. This is larger for larger $M_{\text {sam }}=2048$, and this error increases with the propagation distance even at $\left(M_{\text {sam }}, M_{\text {stop }}\right)=(2048,400000)$. This tendency is relaxed for smaller $M_{\text {sam }}$, but the maximum size of the frequency domain by $M_{\text {sam }}=1024$ is too small to cover the whole intensity spectrum broadened by the nonlinearity as much as in the present case.

It is concluded that the FDM using $\left(M_{\text {sam }}, M_{\text {stp }}\right)$ $=(2048,80000)$ is sufficiently stable, accurate, and efficient.

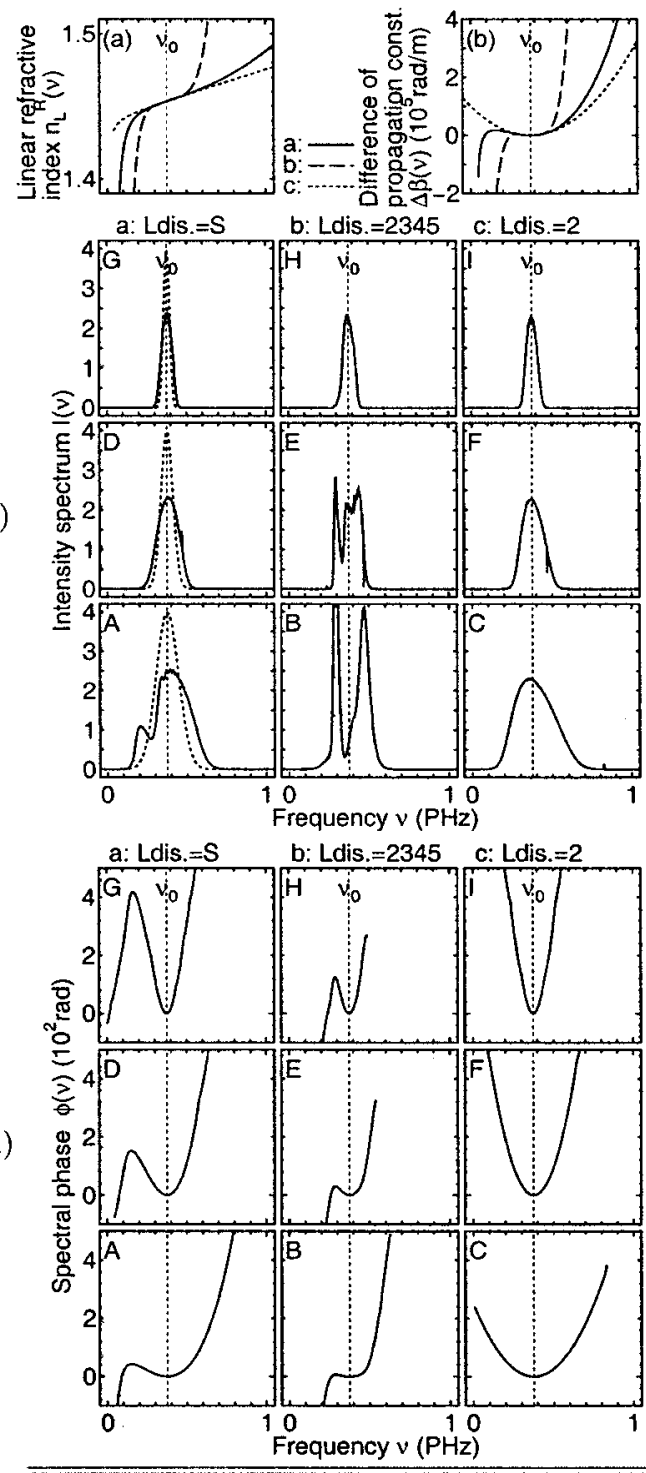

\begin{tabular}{lllllll}
\hline Fig. Cs. Ldis. $\begin{array}{c}P \\
(\mathrm{~kW})\end{array}$ & $N_{\mathrm{sol}}$ & $t_{1}$ & $L_{\mathrm{LD}}$ & $L_{\mathrm{N}}$ & $L_{\mathrm{S}}$ & $z_{\max }$ \\
$(\mathrm{fs})$ & $(\mathrm{mm})$ & $(\mathrm{mm})$ & $(\mathrm{mm})$ & $(\mathrm{mm})$ \\
\hline
\end{tabular}

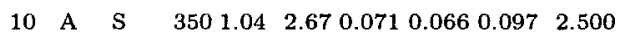

$\begin{array}{lllllllllll}10 & \text { B } & 2345 & 350 & 1.04 & 2.67 & 0.071 & 0.066 & 0.097 & 2.500\end{array}$

$\begin{array}{llllllllllll}10 & \mathrm{C} & 2 & 350 & 1.04 & 2.67 & 0.071 & 0.066 & 0.097 & 2.500\end{array}$

(e)

$\begin{array}{llllllllll}10 & D & S & 100 & 1.04 & 5.00 & 0.250 & 0.231 & 0.637 & 8.803\end{array}$

$\begin{array}{llllllllll}10 & \text { E } & 2345 & 100 & 1.04 & 5.00 & 0.250 & 0.231 & 0.637 & 8.803\end{array}$

$\begin{array}{llllllllll}10 & F & 2 & 100 & 1.04 & 5.00 & 0.250 & 0.231 & 0.637 & 8.803\end{array}$

10 G S 251.0410 .000 .9990 .9235 .09535 .176

$10 \mathrm{H} 2345 \quad 251.0410 .00 \quad 0.9990 .9235 .09535 .176$

$10 \quad \mathrm{I} \quad 2 \quad 251.0410 .000 .9990 .9235 .09535 .176$

FIG. 10. Effect of the linear dispersion keeping the soliton order 1.04 and $\xi=z / L_{\mathrm{LD}}=35.2$ the same: $a$, by the modified Sellmeier equation [8] (Ldis. $=\mathrm{S}$ ); $b$ or $c$, by the propagation constant $\beta(\nu)$ approximated up to the fifth order or the second order in $\nu-\nu_{0}$ (Ldis. $=2345$ or 2). (a) Linear refractive index $n_{\mathrm{L}}^{\mathrm{R}}(\nu)$. (b) Difference of the propagation constant $\Delta \beta(\nu)=\beta(\nu)-\beta\left(\nu_{0}\right)$. (c) Intensity spectrum (dotted curves in $A, D$, and $G$ are initial intensity spectra). (d) Spectral phase. (e) Refer to Table I for headings and abbreviations. 


\section{APPENDIX C: EFFECT OF THE LINEAR DISPERSION}

We observe here how the linear dispersion affects the intensity spectrum $I(\nu)$ and the spectral phase $\phi(\nu)$. Figure 10(a) shows the linear refractive index $n_{\mathrm{L}}^{\mathrm{R}}(\omega)$ [accordingly $n_{\mathrm{L}}^{\mathrm{R}}(\nu)$ ] calculated from the modified Sellmeier equation (16) proposed by Kalosha and Herrmann [8] ( $a$, solid line), or from the propagation constant $\beta(\omega)$ [accordingly $\beta(\nu)$ ] approximated up to the fifth order ( $b$, dashed line) or to the second order ( $c$, dotted line) in $\omega-\omega_{0}$. Figure 10(b) shows the difference of the propagation constant from its value at the central frequency $\Delta \beta(\nu)=\beta(\nu)-\beta\left(\nu_{0}\right)$. Equation (12c) relates $n_{\mathrm{L}}(\omega)$ and $\beta(\omega)$.

Figures 10(c) and 10(d) show the intensity spectra $I(\nu)$ and the spectral phases $\phi(\nu)$ by different linear dispersions and peak powers. As seen from Table I and Fig. 10(e), the central wavelength is commonly $\lambda_{0}=800 \mathrm{~nm}$, the envelope of the initial temporal electric-field profile is Gaussian, and we assume the nonlinearity which is instantaneous and has only the self-steepening as the dispersion, to keep the conditions other than the linear dispersion simple. The initial peak power, initial pulse width, and the maximum fiber length range $P=25-350 \mathrm{~kW}, t_{1}=2.67-10.00 \mathrm{fs}$, and $z_{\max }=2.5-$ $35 \mathrm{~mm}$, respectively, keeping the soliton order 1.04 and $\xi$ $=z_{\max } / L_{\mathrm{LD}}=35.2$ the same, where $z_{\max }$ and $L_{\mathrm{LD}}$ are the maximum fiber length and the dispersion length. Thus, $A, B$, and $C$ are the cases of intense initial peak power, short initial pulse width, and short fiber length.
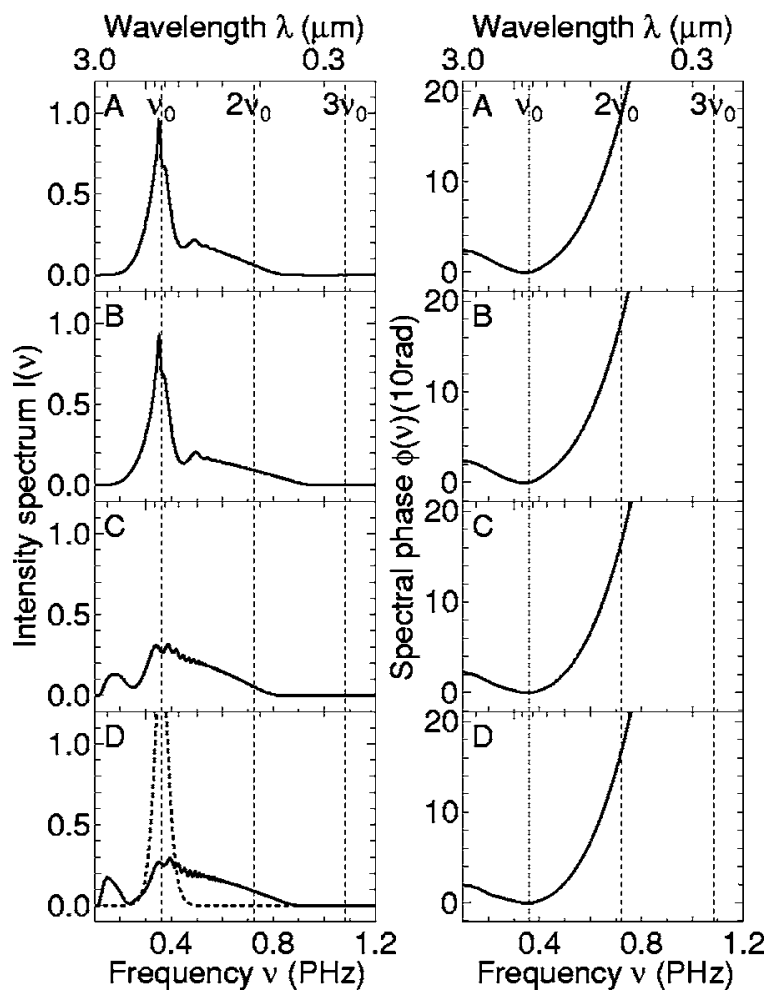

FIG. 11. Combined effects of the delayed Raman response (D), instantaneous response (I), core dispersion (SE), and the flat core dispersion (SF) shown by intensity spectrum (left) and spectral phase (right) at $z=1.0 \mathrm{~mm}$ under the condition of [8]: $A$, by (D)(SE); $B$, by (I)-(SE); $C$, by (D)-(SF); and $D$, by (I)-(SF). The dotted curve with $D$ shows the initial spectrum.
The intensity spectra obtained by the higher-order dispersion up to the fifth order ( $b$, center) are totally different from those in the Sellmeier case ( $a$, left) and those obtained by the quadratic dispersion ( $c$, right). The main peak splits into sharp subpeaks without the growth of wings. On the other hand, the intensity spectra in $c$ approximate those in $a$ rather better than those in $b$.

Though $\xi$ are kept same and the nonlinearity and the linear dispersion almost balance in all cases, we observe broader $I(\nu)$ in $A, B$, and $C$ than those in $G, H$, and $I$ [Fig. $10(\mathrm{c})]$. On the other hand, $\phi(\nu)$ changes with increasing $\left|\nu-\nu_{0}\right|$ more remarkably in $G, H$, and $I$ [Fig. 10(d)]. Thus, $I(\nu)$ seems to be influenced by the nonlinearity rather than by the linear dispersion and the long propagation distance, and vice versa for $\phi(\nu)$. The nonlinear effect other than the SPM, the self-steepening now, possibly affects this difference.

In Fig. 10(d), $\phi(\nu)$, which are obtained by different $\beta$, are similar close to the central frequency $\nu_{0}$, and almost symmetric with respect to $\nu_{0}$. But far from $\nu_{0}$, they are asymmetric other than $\phi(\nu)$ obtained by $\beta$ with the second-order dispersion $(c$, right), and $\phi(\nu)$ by $\beta$ up to the fifth order $(b$, center) is totally different from the others. In the lower frequency range, the spectral phase $\phi(\nu)$ by the modified Sellmeier equation $(a$, left $)$ reaches an extremum, where the group velocity vanishes. As the result of the resonance, a satellite

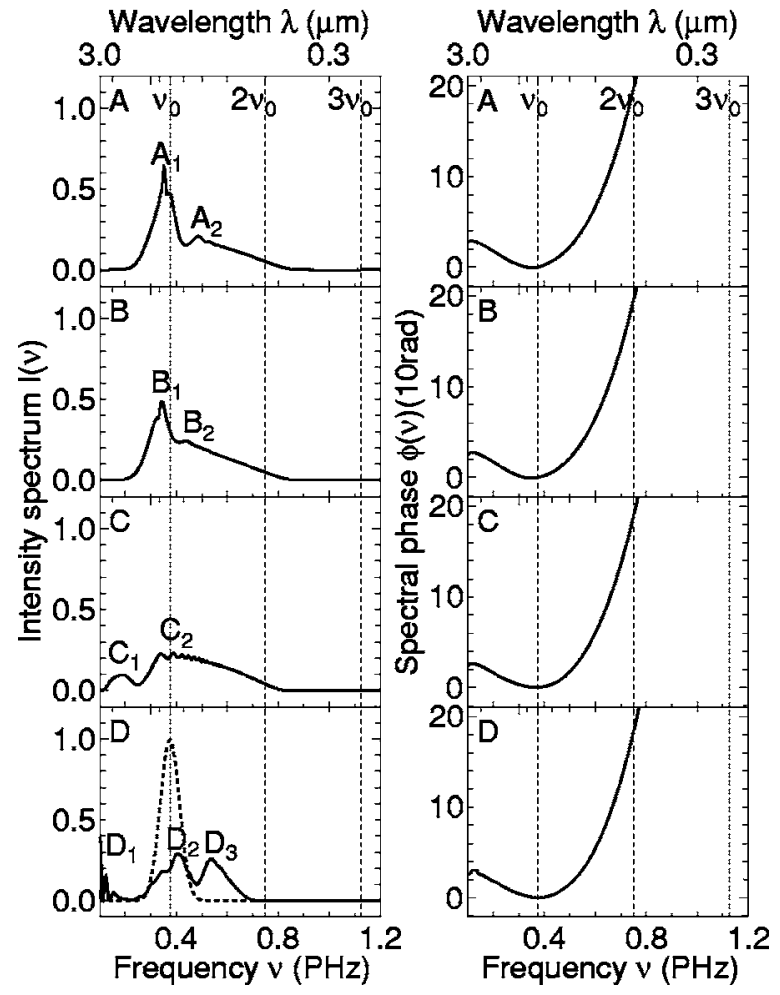

FIG. 12. Effects of nonlinear dispersions (self-steepening, core dispersion) shown by intensity spectrum (left) and spectral phase (right) at $z=1.0 \mathrm{~mm}: A$, by the experimental core dispersion and the self-steepening (SE); $B$, by the theoretical core dispersion and the self-steepening (ST); $C$, by the flat core dispersion and only the self-steepening (SF); and $D$, by neither the core dispersion nor the self-steepening (F). The dotted curve with $D$ shows the initial spectrum. 
appears at this frequency on $I(\nu)$ if $I(\nu)$ is high enough there, as in the case of $A$.

For short pulses we consider now, it is inappropriate to use the propagation constant $\beta(\omega)$ approximated by a polynomial in $\omega-\omega_{0}$. Within the width of the spectrum, it leaves far away from the correct linear dispersion.

\section{APPENDIX D: COMBINED EFFECTS OF THE DELAYED RAMAN RESPONSE AND NONLINEAR DISPERSIONS}

Combined effects of the delayed Raman response and the core dispersion are investigated in Fig. 11, under the same set of quantitative conditions as Kalosha-Herrmann (hereafter K-H) [8], $\lambda_{0}=830 \mathrm{~nm},\left(P, t_{1}\right)=(2290 \mathrm{~kW}, 5.00 \mathrm{fs})$, and $z=1.0 \mathrm{~mm}$. The spectrum $I(\nu)$ of $D$ corresponds to that obtained in [8] with the instantaneous response and the flat core dispersion. Comparisons between the delayed Raman response and the instantaneous response- $A$ and $B$ or $C$ and $D$ of Fig. 11-show that the effect of the delayed Raman response is small whether with or without the experimental core dispersion. As seen from Fig. 1(b), the imaginary part of $\widetilde{R}(\omega)$ causing the effect of the delayed Raman response almost vanishes outside of the narrow range between $\omega_{\mathrm{S}}$ and $\omega_{\mathrm{A}}$. Thus, only the slight deformation of the spectrum is observed instead of the self-frequency shift under the present condition of the broad initial spectrum and the short propagation distance. On the other hand, after the core dispersion is introduced $(A, B)$, the satellite in the lower frequency dis- appears, the main peak sharpens, the subpeak in the higher frequency appears, and the wing stretches. These effects are caused by the nonlinear effects decreased in the lower frequency range and increased in the higher frequency range by the core dispersion. On the other hand, the spectral phase on the right side of Fig. 11 is rather insensitive to these effects.

Figure 12 compares $I(\nu)$ for different nonlinear dispersions (self-steepening, core dispersion). The delayed Raman response is taken into account in all of them. In $D$ with no nonlinear dispersion, there are three peaks around $0.12,0.43$, and $0.54 \mathrm{PHz}\left(D_{1}, D_{2}\right.$, and $\left.D_{3}\right)$, among which $D_{2}$ and $D_{3}$ are above $\nu_{0}$. From $D$ to $C$, only the self-steepening increasing proportionally to the frequency is added. Then, $D_{1}$ becomes a broad satellite around $0.19 \mathrm{PHz}\left(C_{1}\right)$, and $D_{2}, D_{3}$ merge into one peak around $0.35 \mathrm{PHz}\left(C_{2}\right)$ with a broad wing in the high frequency range. From $C$ to $B$, the theoretical core dispersion is introduced, which weakens the nonlinearity in the lower frequency range and strengthens it in the higher frequency range more. Owing to this, $C_{1}$ vanishes, $C_{2}$ sharpens into a main peak $\left(B_{1}\right)$ without the shift of the position, and a gentle shoulder appears around $0.43 \mathrm{PHz}\left(B_{2}\right)$. From $B$ to $A$, the core dispersion is replaced with the experimental one, where the core radius above $\nu_{0}$ is slightly larger (below $\lambda_{0}$ in the inset in Fig. 2). Then, $B_{2}$ is recognized as a subpeak caused by the relaxed flow of the spectral components from $\nu_{0}$ toward the higher frequency range. In the nonlinear dispersions, the self-steepening forms the basic structure of $I(\nu)$, and the core dispersion reforms it.
[1] K. Yamane, Z. Zhang, K. Oka, R. Morita, and M. Yamashita, Opt. Lett. 28, 2258 (2003).

[2] K. Yamane, T. Naoi, A. Suguro, R. Morita, and M. Yamashita, in Technical Digest of Conference on Lasers and ElectroOptics (CLEO) / International Quantum Electronics Conference (IQEC) (Optical Society of America, Washington, 2005), JThE18.

[3] K. Yamane, T. Kito, R. Morita, and M. Yamashita, in Technical Digest of Conference on Lasers and Electro-Optics (CLEO) / International Quantum Electronics Conference (IQEC) (Optical Society of America, Washington, 2004), Postdeadline Paper Book CPDP2.

[4] D. N. Christodoulides and R. I. Joseph, Appl. Phys. Lett. 47, 76 (1985).

[5] T. Brabec and F. Krausz, Phys. Rev. Lett. 78, 3282 (1997).

[6] K. E. Oughstrun and G. C. Sherman, J. Opt. Soc. Am. B 5, 817 (1988).

[7] S. Shen and K. E. Oughstrun, J. Opt. Soc. Am. B 6, 948 (1989).

[8] V. P. Kalosha and J. Herrmann, Phys. Rev. A 62, 011804(R) (2000).

[9] K. J. Blow and D. Wood, IEEE J. Quantum Electron. 25, 2665 (1989).

[10] R. H. Stolen, J. P. Gordon, W. J. Tomlinson, and H. A. Haus, J. Opt. Soc. Am. B 6, 1159 (1989).

[11] P. V. Mamyshev and S. V. Chernikov, Opt. Lett. 15, 1076 (1990).

[12] S. V. Chernikov and P. V. Mamyshev, J. Opt. Soc. Am. B 8,
1633 (1991).

[13] R. M. Joseph, S. C. Hagness, and A. Taflove, Opt. Lett. 16, 1412 (1991).

[14] P. M. Goorjian, A. Taflove, R. M. Joseph, and S. C. Hagness, IEEE J. Quantum Electron. 28, 2416 (1992).

[15] P. M. Goorjian and A. Taflove, Opt. Lett. 17, 180 (1992).

[16] H. Xiao and K. E. Oughstrun, J. Opt. Soc. Am. B 16, 1773 (1999).

[17] R. H. Hardin and F. D. Tappert, SIAM Rev. 15, 423 (1973).

[18] R. A. Fisher and W. K. Bischel, Appl. Phys. Lett. 23, 661 (1973).

[19] R. A. Fisher and W. K. Bischel, J. Appl. Phys. 46, 4921 (1975).

[20] G. P. Agrawal, Nonlinear Fiber Optics (Academic Press, San Diego, 2001), Chap. 2.

[21] R. H. Stolen and C. Lin, Phys. Rev. A 17, 1448 (1978).

[22] A. Baltuska, Z. Wei, M. S. Pshenichnikov, and D. A. Wiersma, Opt. Lett. 22, 102 (1997).

[23] N. Karasawa, S. Nakamura, N. Nakagawa, M. Shibata, R. Morita, H. Shigekawa, and M. Yamashita, IEEE J. Quantum Electron. 37, 398 (2001).

[24] S. Nakamura, L. Li, N. Karasawa, R. Morita, H. Shigekawa, and M. Yamashita, Jpn. J. Appl. Phys., Part 1 41, 1369 (2002).

[25] D. Marcuse, J. Opt. Soc. Am. 68, 103 (1978).

[26] A. Apolonski, A. Poppe, G. Tempea, C. Spielmann, T. Udem, R. Holzwarth, T. W. Hansch, and F. Krausz, Phys. Rev. Lett. 85, 740 (2000). 